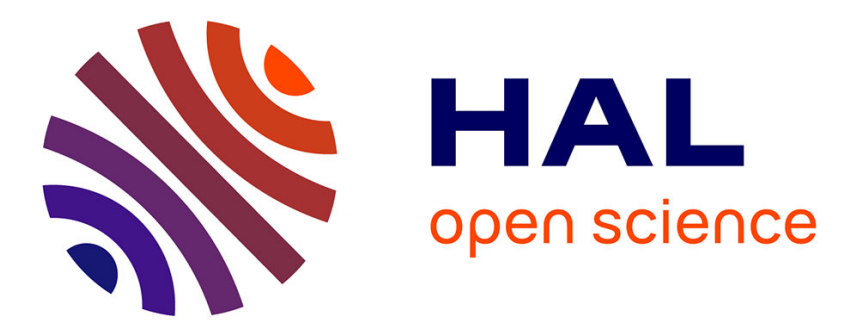

\title{
Electroencephalography (EEG)-based Brain-Computer Interfaces
}

Fabien Lotte, Laurent Bougrain, Maureen Clerc

\section{To cite this version:}

Fabien Lotte, Laurent Bougrain, Maureen Clerc. Electroencephalography (EEG)-based BrainComputer Interfaces. Wiley Encyclopedia of Electrical and Electronics Engineering, Wiley, pp.44, 2015, 10.1002/047134608X.W8278 . hal-01167515

\section{HAL Id: hal-01167515 https://hal.inria.fr/hal-01167515}

Submitted on 24 Jun 2015

HAL is a multi-disciplinary open access archive for the deposit and dissemination of scientific research documents, whether they are published or not. The documents may come from teaching and research institutions in France or abroad, or from public or private research centers.
L'archive ouverte pluridisciplinaire HAL, est destinée au dépôt et à la diffusion de documents scientifiques de niveau recherche, publiés ou non, émanant des établissements d'enseignement et de recherche français ou étrangers, des laboratoires publics ou privés. 


\title{
Electroencephalography (EEG)-based Brain-Computer Interfaces
}

\author{
Fabien LOTTE ${ }^{1}$, Laurent BOUGRAIN ${ }^{2}$, Maureen CLERC ${ }^{3}$ \\ ${ }^{1}$ Inria Bordeaux Sud-Ouest, FRANCE \\ ${ }^{2}$ Lorraine University/Inria Nancy Grand-Est, FRANCE \\ ${ }^{3}$ Inria Sophia Antipolis Méditerranée, FRANCE
}

June 1, 2015

\begin{abstract}
Brain-Computer Interfaces (BCI) are systems that can translate the brain activity patterns of a user into messages or commands for an interactive application. The brain activity which is processed by the BCI systems is usually measured using Electroencephalography (EEG). In this article, we aim at providing an accessible and up-to-date overview of EEG-based BCI, with a main focus on its engineering aspects. We notably introduce some basic neuroscience background, and explain how to design an EEG-based BCI, in particular reviewing which signal processing, machine learning, software and hardware tools to use. We present Brain Computer Interface applications, highlight some limitations of current systems and suggest some perspectives for the field.
\end{abstract}

\section{Introduction}

Since the first experiments of Electroencephalography (EEG) on humans by Hans Berger in 1929 [1], the idea that brain activity could be used as a communication channel rapidly emerged. EEG is a technique which measures, on the scalp and in real-time, small electrical currents that reflect brain activity. As such, EEG discovery has enabled researchers to measure brain activity in humans and to start trying to decode this activity. After some attempts by music composers to use brain activity to produce music in real time $[2,3]$, the concept of a Brain-Computer Interface (BCI) was formalised in 1973 by Vidal [4]. A BCI is a system that translates the brain activity patterns of a user into messages or commands for an interactive application, this activity being measured and processed by the system $[5,6]$. For instance, a BCI could enable a user to move a cursor on a screen left or right by imagining left or right hand movements, respectively. Since the design of the first real-time BCI in the 90's $[7,8,9,10]$, the BCI field has grown tremendously, involving hundreds of laboratories and companies around the world, and becoming a more mature field of research and 
technology $[11,6]$. BCI have indeed evolved to become more robust, more accessible and promising for a wide range of applications including communication and control for motor impaired users [9, 12], gaming targeted toward the general public [13, 14], real-time mental state monitoring [15] or stroke rehabilitation $[16,17]$.

Designing a BCI is a complex and difficult task which requires multidisciplinary knowledge in computer science, engineering, signal processing, neuroscience and psychology. In order to use a BCI, two phases are generally required: 1) an offline training phase during which the system is calibrated and 2) the operational online phase in which the system recognises brain activity patterns and translates them into commands for a computer. An online BCI system is a closed-loop, generally composed of six main steps: brain activity measurement, preprocessing, feature extraction, classification, translation into a command and feedback:

1. Brain activity measurement allows to acquire the raw signals reflecting the user's brain activity [18]. Various types of sensors can be employed, but in this article we focus on EEG as the measurement technique.

2. Preprocessing consists in cleaning and denoising input data in order to enhance the relevant information contained in the raw signals [19].

3. Feature extraction aims to describe the signals by a few relevant values called "features" [19]. These features can be, for instance, the power of the EEG over selected channels, and in specific frequency bands.

4. Classification assigns a class to a set of features extracted from the signals within a certain time range [20]. This class corresponds to the kind of brain activity pattern identified (e.g., imagined left hand movement or imagined right hand movement). Classification algorithms are known as "classifiers".

5. Translation into a command associates a command to the brain activity pattern identified in the user's brain signals, e.g., a recognised left hand movement could be translated into the command "move the cursor left". This command is then used to control a given application such as a speller (text editor, see part 4.1) or a robot [21].

6. Feedback is provided to the user to inform him/her about the recognised brain activity pattern. This aims to help the user modulate his/her brain activity and as such improve his/her control over the BCI [5]. Indeed, BCI is a skill that needs to be learned and refined [22].

This whole architecture is summarised in Figure 1. These steps define an online BCI. The BCI research community is in quest of solutions to avoid a time-consuming calibration stage before operating a BCI (see, e.g., [23, 24]). Currently, calibration is generally necessary in order to obtain a reliable BCI operation, and is generally done offline. In this stage, the classification algorithm 
is calibrated and the optimal features, and relevant sensors are selected. For this calibration, a training data set needs to be prerecorded from the user. Indeed, EEG signals are highly user-specific, and as such, most current BCI systems are calibrated specifically for each user. This training data set should contain EEG signals recorded while the user performed each mental task of interest several times, according to given instructions. The recorded EEG signals will be used as mental state examples in order to find the best calibration parameters for this user.

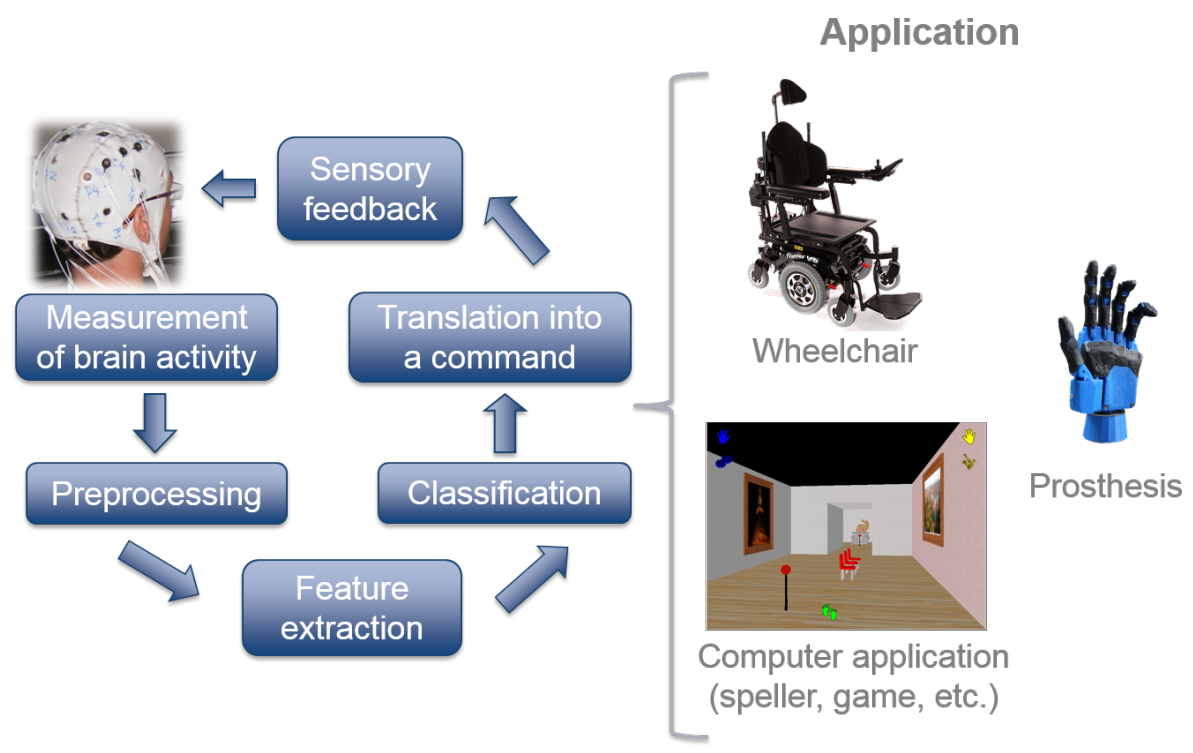

Figure 1: General architecture of an online brain-computer interface, with examples of applications.

It should be noted that, although BCI are a very promising technology, they still suffer from a number of limitations that need to be overcome before they can be used in practical applications, outside laboratories. Among these, we can mention the relatively modest robustness and reliability of such systems. Indeed, most BCI very rarely yield $100 \%$ correct recognition of the user's mental commands [25, 26, 27], and some users are completely unable to use a given type of BCI [28]. Moreover, while current EEG-based BCI are reasonably stable in laboratory conditions, their performance decreases significantly when confronted to real-word, complex environments, over long periods, or when the user is moving [29, 30, 31]. BCI reliability is also much lower when used by severely motor-impaired users such as locked-in patients [32]. EEG-based BCI also generally require relatively long calibration time [24] and may also require long to very long human training time $[22,33]$. A number of challenges therefore still need to be tackled by the research community to yield robust, practical 
EEG-based BCI.

In this article, we aim at providing an accessible and up-to-date overview of EEG-based BCI (EEG being by far the most used modality for BCI design), with a main focus on its engineering aspects. After a brief presentation of some basic BCI-related neuroscience background (Section 2), Section 3 describes how to design an EEG-based BCI, and notably how to process and classify EEG signals. Then, Section 4 presents the main current applications of BCI technologies, while Section 5 details the main hardware and software components available to design these BCI. Finally, Section 6 concludes the article with a discussion on the future of BCI research and technology.

\section{Some brief neuroscience background}

At the centre of the BCI loop is the user and his/her brain, whose activity is being measured and processed by the BCI. In this section, we provide some brief notion of brain anatomy relevant for BCI and briefly describe how to measure brain activity and what kind of brain activity patterns can be detected and used by the BCI.

\subsection{Anatomy and brain imaging}

The brain includes three main parts: the cerebellum, the brainstem and the cerebrum (which includes the cortex). The cortex is composed of the right and left cerebral hemispheres, each of which being subdivided into four lobes: frontal, parietal, temporal and occipital. The central fissure (or central sulcus) separates the parietal lobe from the frontal lobe, and the primary motor cortex from the primary somatosensory cortex. The frontal lobe is associated with motor learning, problem solving, language, reasoning, planning, memory, and is the core for personality and emotions. The primary motor cortex, located in this lobe, is associated with motor activity, for planning and executing movements. Broadly speaking, the muscles to the left of the body are controlled by the right hemisphere, and the left hemisphere controls the right side. The primary motor cortex sends information needed to perform voluntary movements and receive sensory information from other brain areas. The received sensory information is used as feedback to plan and execute movements. Furthermore, the primary motor cortex contains a broad representation of the anatomy, see Figure 2, where different body parts are partially controlled by overlapping regions of the motor cortex.

The parietal lobe plays an important role in the perception of tactile stimuli: it responds to sensory inputs, such as temperature, pressure and pain. It is also associated with movement coordination and language. The occipital lobe is responsible for the early stages of processing of visual stimuli and spatial recognition. The temporal lobe plays an important role in the perception of auditory and olfactory stimuli. It is also associated with coordination and balance, emo- 
tions, memory and face recognition.

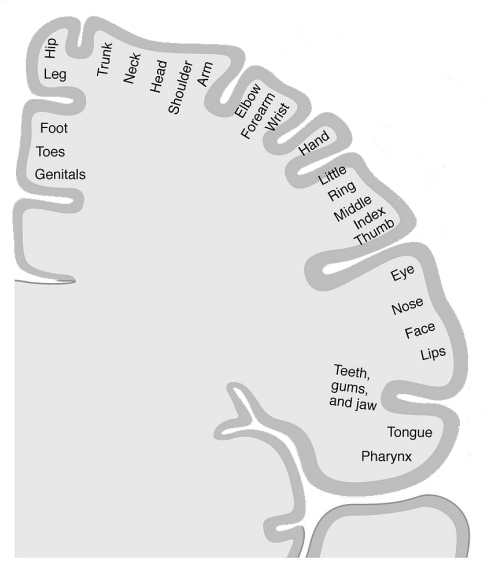

Figure 2: Primary motor cortex of the right hemisphere: point-by-point correspondence of a body area with a specific area of the primary motor cortex. The central rectangle of Figure 3 indicates the corresponding locations on EEG electrodes.

There are several systems for recording brain activity. They can be classified i) by the location at which the signal is measured (invasive vs non-invasive) and ii) by the type of sensors used to record the brain signals. These sensors can measure electric, magnetic or chemical signals. BCI based on invasive recordings such as electrocorticography and intracortical electrodes are mainly performed with animals and human patients implanted for clinical diagnosis. Non-invasive technologies, which do not penetrate the skin, do not represent any risk for the user, such as, haemorrhages, infections or bioincompatibility. Some of the available non-invasive techniques include functional magnetic resonance imaging (fMRI) and functional near infrared spectroscopy (fNIRS) - sensitive to blood oxygenation - positron emission tomography (PET) and single photon emission computed tomography (SPECT) - based on nuclear medicine - , transcranial Doppler ultrasound (TcD) - based on ultrasound - and finally magnetoencephalography (MEG) and electroencephalography (EEG) - directly measuring electromagnetic fields generated by neuronal electrical activity.

EEG is the most common method for recording brain signals in humans because it is safe, affordable, easy to use, and because it has a high temporal resolution (of the order of milliseconds) [34]. EEG electrodes, placed on the scalp, can be either "passive" or "active". Passive electrodes, which are metallic, are connected to an amplifier by a cable. Active electrodes have an inbuilt preamplifier to make them less sensitive to environmental noise and cable movements. Both types of electrodes need gel or saline liquid to operate, in order 
to reduce the skin-electrode contact impedance. This prevents their use for long periods, such as a whole day, because the gel dries with time, causing the impedances to rise and the signals to degrade. For this reason, dry electrodes are currently being developed and tested for BCI (see Section 5). Despite a low spatial resolution (at best the inter-distance between electrodes is 1-2 cm) and a low signal-to-noise ratio, this solution presents many advantages for widespread use.

In early recordings of brain activity using EEG, there was no rule concerning the layout of the electrodes on the head. Then common protocols were defined to standardise the layout, the first one being the 10-20 system [35], still used today. The name of the system comes from the inter-electrode distances, which are approximately $10 \%$ or $20 \%$ of the front-to-back or right-to-left head perimeters (see Figure 3).

An amplifier is necessary for acquiring measurable signals. Modern amplifiers produce a set of digital signals, one per channel. Each channel measures the electric potential difference between the electrode to which it is connected and a reference electrode. This reference electrode can be placed anywhere on the head, but should not be at a location where the activity is very strong, because it could prevent weaker activity of other regions from being correctly measured. Many positions can be selected as reference, for instance an earlobe, or the mastoid region behind the ear. Some amplifiers apply a common average reference, by subtracting from each channel the average potential over all channels, a good strategy when recording from a large number of electrodes (so called high-density EEG). A ground electrode is also necessary to prevent power

line interference with the low-amplitude signals of interest. This ground can be placed anywhere on the body, and it is sometimes also placed on the scalp.

The next sections describe the three main brain activity patterns that are used to design EEG-based BCI: Event Related Desynchronization/Synchronization, Event Related Potentials and Steady State Evoked Potentials. Note that these are not the only existing brain activity patterns used in EEG-based BCI, but only the most popular and so far the most efficient for communication and control applications.

\subsection{Event Related Desynchronization/Synchronization}

Event-Related Desynchronization or Synchronization (ERD/ERS), corresponds to amplitude variations of EEG signals oscillations, i.e., amplitude changes in the power of EEG signals in certain frequency bands [36]. ERD corresponds to thousands or millions of cortical neurons firing in a more desynchronised way than in the baseline (rest condition where no event is occurring). This results in a smaller amplitude oscillations in scalp EEG signals. On the contrary, ERS corresponds to cortical neurons firing synchronously, hence leading to a resulting large amplitude of scalp EEG signals. More formally, according to Pfurstcheller et al, ERD and ERS can "be considered to be due to a decrease or an increase in synchrony of the underlying neuronal populations" respectively [36]. ERD 


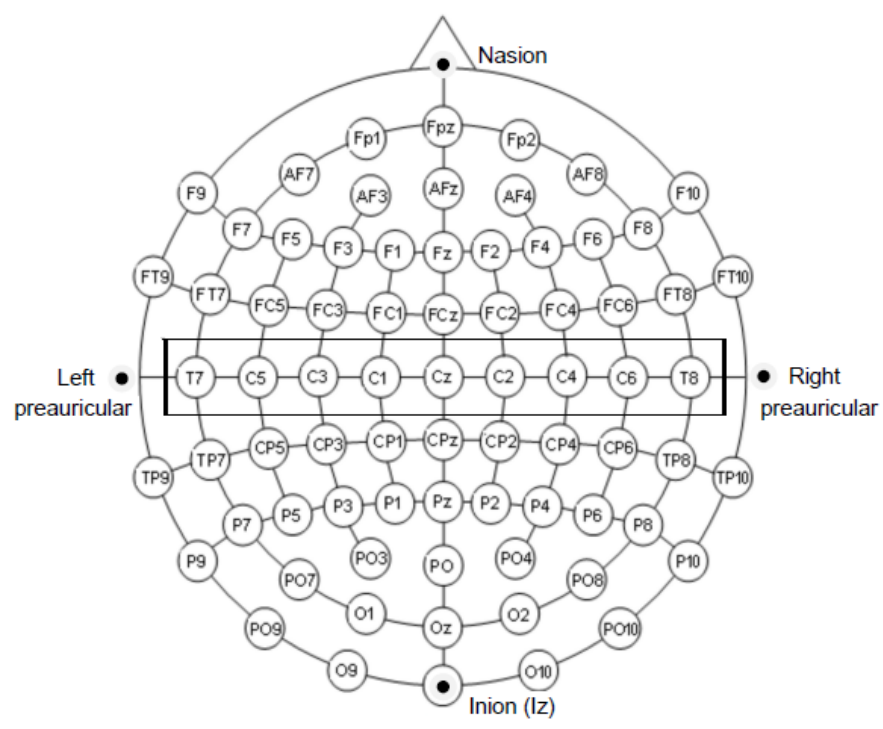

Figure 3: Extended 10-20 EEG system. Electrodes are identified with labels: the letters correspond to cortical locations: frontal $(\mathrm{F})$, temporal $(\mathrm{T})$, parietal $(\mathrm{P})$, occipital $(\mathrm{O})$, central $(\mathrm{C})$ and combinations of these to indicate intermediate locations ( $\mathrm{Fp}$ means frontal polar and corresponds to the anterior pre-frontal lobe). The electrode numbers indicate laterality: odd numbers to the left, even numbers to the right, and the letter " $\mathrm{z}$ " (meaning zero) on the midline. The electrode layout displayed considers $10 \%$ intermediate distances. The central rectangle indicates the location of the primary motor cortex (adapted with the permission of gtec medical engineering $\mathrm{GmbH}$ ).

and ERS have been suggested to reflect cortical activation and deactivation, respectively [36].

ERD/ERS can be observed during a number of different mental tasks, and can thus be used as a feature to drive a BCI. In particular, ERD/ERS can be observed during Motor Imagery (MI), as well as executed movement [37] (see, e.g., Figure 7). For instance, an execution or an imagination of a left-hand movement leads to an ERD in the $\mu(8-12 \mathrm{~Hz})$ and $\beta(16-24 \mathrm{~Hz})$ bands (the socalled sensorimotor rhythms) during movement, and to an ERS in the $\beta$ band after movement. Both the ERD and the ERS occur in the controlateral sensorimotor cortex (in the hemisphere opposite to the hand used). ERD/ERS can also be observed during diverse mental imagery tasks, such as mental rotation of geometric figures or mental subtractions, among others [38, 39].

ERD/ERS-based BCI have three main advantages: 1) they can be rather natural and intuitive to use, since their users "just" have to imagine a specific task to send control commands, 2) they do not require any stimulus (contrary to ERP-based BCI, see Section 2.3), which enables the users to devote all their 
sensory attention to the feedback of the BCI and/or to their external environment 3) they can be used in a self-paced way, that is, the user can initiate the task (and hence the command) at will. Their main drawback is the fact that such BCI require substantial training on the user side, to find the correct imagination strategy that leads to the clearest and most distinct ERD/ERS. Also, this kind of BCI can currently only be used with a maximum of 3 or 4 different mental imagery tasks to ensure maximal performances: with more tasks, their recognition accuracy becomes considerably degraded, and therefore the overall BCI efficiency decreased [40].

\subsection{Event Related Potentials}

Event Related Potentials (ERPs) are stereotyped deflections of the EEG, related to a sensory, cognitive or motor event. The "Event" of an ERP must come at a precise measurable time, called the time trigger of the event. Typically, if a person is receiving a sensory stimulus, ths time trigger is the instant at which the stimulus is presented, or, in case of a button press, it is the instant at which the muscular activity exceeds a certain threshold. The deflections measured by the ERPs are represented as waveforms, and sometimes called waves. But in spite of this terminology, ERPs do not display sustained oscillations, contrarily to the ERD/ERS of part 2.2.

ERP nomenclatures are very varied: they can indicate their context of occurrence (Somatosensory Evoked potential (SEP) - in response to a tactile stimulation; Error Potential (ErrP) - related to an error), or the polarity of the deflection: negativity indicating a decrease in amplitude, and positivity an increase in amplitude. For instance, the Mismatch Negativity (MMN) - is a negative deflection of the EEG in response to a deviant stimulus within a sequence of repeating stimuli, either auditory or visual [41]. The timing of the deflection is indicative of the level of processing involved: very early components (20 to $100 \mathrm{~ms}$ after the event trigger) are automatic, reflex responses whereas later deflections occurring after $200 \mathrm{~ms}$, reflect a higher-level, cognitive, processing. This timing is also often part of ERP nomenclatures: an auditory N100 or visual $N 1$ are early negative deflections that may be modulated by attention; the P300 wave - positive deflection, occurring approximately $300 \mathrm{~ms}$ after the presentation of a deviant stimulus to which the person is paying attention.

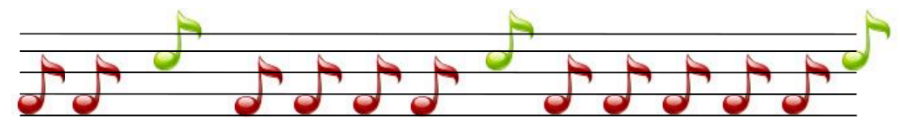

Figure 4: An auditory oddball sequence: the deviant sounds (high-pitched, in green) are presented in a stream of standards (low-pitched, in red). A P300 wave occurs after the deviant sounds. When a person is asked to actively attend to the deviants, e.g. by counting their occurrences, the P300 wave is stronger than if the auditory stream is only passively attended to. 
Although not reflected in their nomenclature, spatial locations of ERPs are sterotypical. For instance, the P300 wave is similar to the Mismatch Negativity because both are produced by an "oddball" effect (see Figure 4), and they both occur at similar latencies. The (negative) MMN would be canceled out by the (positive) P300 (which has higher amplitude) if they were not located at different regions on the scalp: the P300 location is parieto-occipital, whereas the MMN occurs on occipital (visual stimuli) or fronto-central (auditory stimuli) electrodes. We next present in more detail the two ERPs the most exploited in BCI at present: the P300 wave, and the Error Potential.

The P300 wave is elicited by an oddball sequence of auditory or visual stimuli [42]. The waveform rises and wanes between 250 and $500 \mathrm{~ms}$ after the occurrence of a deviant within a stream of standard stimuli, to which the user is paying attention (for instance, by counting the number of deviants in the stream). An example of a P300 can be seen in Figure 10. The P300 wave is in fact composed of two distinct subcomponents, the P3a (fronto-central, related to automatic detection of novelty) and the P3b (centro-parietal, related to the voluntary detection of targets) [43].

Auditory as well as visual oddball stimulation paradigms have been used to develop P300-based Brain Computer Interfaces. Interestingly, the P300 wave is also elicited when the stimulation stream contains more than two items, only one of which is being actively attended to at a given time. The BCI applications therefore consist of forced-choice tasks: the system detects the sequence of items to which the user is paying attention. Section 4.1 describes in more detail the main BCI application based on the P300 wave: the P300-speller which allows to type on a virtual keyboard with flashing letters.

Error Potentials arise under several forms: the occurrence of an error produces, 50-100 ms after a motor response, an Error-Related Negativity (Ne, fronto-central), followed by a positive deflection (Pe, centro-parietal). But relying on motor response to obtain the timing of the $\mathrm{Ne} / \mathrm{Pe}$, is not realistic in BCI, where the goal is to bypass muscular commands. Another form of Error Potential, more appropriate for non-muscular BCI control, is the Feedback Related Negativity: it is time-locked to the presentation of a feedback. The FRN occurs when the feedback does not conform with the user's expectations.

The use of Error Potentials in BCI arises from the observation that this additional information provided automatically by the user could be used to improve the BCI performance [44, 45].

There are advantages and drawbacks to using ERPs in BCI. The two main advantages are that 1) ERPs are stereotypical responses, which the user can produce without any particular training; 2) ERPs occur at short latencies, which is a beneficial property for the throughput of a BCI. However, these advantages are counterbalanced by some drawbacks: 1) ERPs have a low amplitude (a 
few tens of $\mu \mathrm{V}$ ), and are dominated by background activity, which makes them difficult to detect; 2) There are large inter-individual differences in ERP latencies and waveforms, requiring the system to be trained to recognize the ERP of a given individual. 3) ERPs are locked to an event occurring at a specific time, so ideally the BCI protocol should provide this type of stimulation, while controlling its precise timing.

\subsection{Steady State Evoked Potentials}

Steady State Evoked Potentials (SSEP) are measured when the subject perceives a periodic stimulus such as a flickering picture or an amplitude-modulated

sound. SSEP are defined by an increase of the EEG power at the frequency of the stimulus (e.g., the flickering frequency of the picture or the modulation frequency of the sound) or at its harmonics or sub-harmonics [46, 47, 27]. Interestingly, SSEP are modulated by attention: the more attention the user pays to a stimulus the higher the SSEP response. As such, if the user is provided with multiple periodic stimulus, each stimulus with a different stimulation frequency, it is possible to detect in the EEG signals which of these stimuli the user is attending to, by finding the largest SSEP response. Each stimulus can be associated to a command, leading to SSEP-based BCI with multiple commands. Various kinds of SSEP are used for BCI, such as Steady State Visual Evoked Potentials (SSVEP) [46, 47], somatosensory SSEP [48] or auditory SSEP [49]. These SSEP can be observed over the brain areas corresponding to the sensory channel being stimulated, such as the visual areas (occipital cortex) when SSVEP are used. SSVEP are by far the most used SSEP for BCI design, and were successfully used in multiple applications such as SSVEP-based BCI wheelchair control [50], spellers [51] or gaming applications [52], among many others.

SSVEP are increasingly popular as they can be used quickly without user training, and can provide a large number of commands. Their main limitations are the need for a stimulus, and the reliance on the user's visual attention.

\subsection{Hybrid BCI}

Interestingly, all the different types of EEG patterns that we have just presented, and that can be used to operate a BCI, do not require to be used in isolation. Multiple such signals can be combined together, e.g., to control a wheelchair based on both P300 and SSVEP [50], or combined with other control signals not originating from the brain, such as eye trackers or joysticks $[53,54]$. Such BCI combining multiple types of control signals are known as Hybrid BCI [55]. Such hybrid BCI approaches are a promising approach to make BCI more robust, by combining multiple inputs and control signals, as well as to extend the target user population of BCI. For instance, by combining EEG signals with signals from muscular activity (electromyogram - EMG), such hybrid BCI could prove useful not only for completely or severely paralysed users (historically, the initial target population of $\mathrm{BCI}$ ) but also for motor-impaired users with residual muscle 
control, e.g. stroke patients. The interested reader can refer to the survey in [56] for more information on the use of Hybrid BCI for clinical applications.

\section{EEG-based BCI Design}

Once the EEG signals acquired, they must be analysed and processed in order to identify the brain activity patterns they contain. As mentioned above, this is achieved by first preprocessing the signals, then extracting features which are then classified. These three steps are detailed below, and followed by a section on sensory feedback, closing the BCI system loop.

\subsection{Preprocessing}

The electrical activity of the brain causes variations of electric potential on the scalp of only a tens of few microVolts. Care must be taken when acquiring the EEG, and when processing the signals, in order for the relevant information to be preserved and revealed.

Part of the EEG signals comes from electrical activity unrelated to the brain: muscular activity of the head and neck, eye movements which are equivalent to rotating electric dipoles, eye blinks which generate electric charge variations (see Figure 5, for examples of such artefacts). Such biosignals can of course provide useful information (indicating discomfort, or attention lapses). One should be aware of these artefacts and be careful not to process them indistinctly from brain signals, for BCI that aims to process information coming strictly from the brain [30].

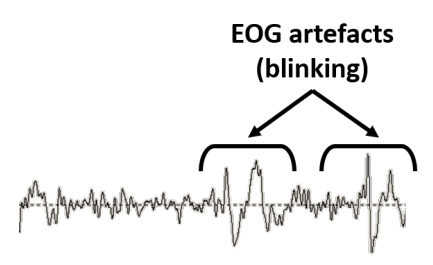

a)

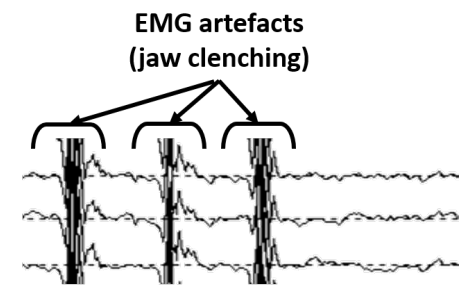

b)

Figure 5: Examples of artefacts polluting EEG signals. a) Electrooculographics (EOG) artefacts due to blinking, b) Electromyographic (EMG) artefacts due to jaw clenching.

Detecting artefacts can be done with amplitude thresholds on bandpower (muscular artefacts) or time-courses of certain channels. For ocular artefacts, additional electrodes can be placed close to the eyes, allowing to recognise a trace of that activity on the EEG channels. 
Once an artefact has been detected, the portion of data corrupted with this artefact should be rejected (i.e. disregarded) in two cases: 1) if the effect of the artefact on the rest of the signal is too strong to be removed; 2) if the artefact indicates that correct conditions are not met for pursuing with the BCI at that particular time. In other cases, one can subtract the effect of the artefact from the EEG by using special techniques such as Independent Components Analysis or specific filters [57].

EEG signals can be represented in three different domains: in time (as they are acquired by the amplifier), in frequency (spectral power) and in space (topographical layout on the scalp according to the electrode positions, as in Figure 3).

Time-based representations are especially useful when dealing with eventrelated potentials (ERPs). ERPs come with time triggers (see 2.3), which allow to extract epochs, which are time-windowed portions of signals. The EEG measures a mixture of activities coming from many regions of the brain, and although ERPs are stereotyped deflections, they are difficult to distinguish in a single epoch. Statistical procedures are called for to extract the significant deflections from several epochs (or trials). The simplest statistical procedure is cross-trial averaging: this decreases the amplitude of the background oscillations, whose phase is not consistent across the trials. For Brain Computer Interfaces where the analysis must be done in real time, there are specific techniques for single-trial detection of ERPs (see 3.2.2).

Frequency-based representations reveal the spectrum of the EEG, and can be applied on sliding windows: such time-frequency representations are necessary for BCI real-time processing. Since relevant brain activity is often frequency-dependent (e.g. ERD/ERS reviewed in part 2.2), it is useful to bandpass filter the signals to the frequency-bands of interest. This also reduces the influence of noise coming from other frequencies, e.g. power-line noise.

Spatial representations reveal spatial patterns, also called topographies, related to the brain areas where the activity is produced. In order to enhance the signal to noise ratio, some spatial processing can be applied to the EEG. Indeed, electric fields on the scalp result from electrical conduction within the head volume. Because the skull is a poor electrical conductor, scalp topographies are blurred compared to the distribution of cortical sources. Source reconstruction, through an inverse problem, allows to pinpoint the activity of interest at the cortical level [58]. BCI features can then be defined in source space [59, 60], allowing to make use of a priori information. For instance, detection of an errorrelated potential is facilitated by only considering the source activity originating from the anterior cingulate cortex. However, rather than source space analysis, simpler spatial filters, known as Laplacian filters, are more commonly used. The Laplacian enhances the SNR of the data, by bringing out the differences between neighboring electrodes. In fact, the Laplacian filter crudely approximates source reconstruction: it performs a deconvolution of the blurring due to volume conduction $[61,62]$. 


\subsection{Feature extraction}

As mentioned before, feature extraction aims at representing raw or preprocessed EEG signals by an ideally small number of relevant values, which describe the task-relevant information contained in the signals. These features should be selected to minimise the intra-class feature variances while maximising interclass variances. In other words, their values should be as different as possible between different classes. BCI based on oscillatory activity (e.g., BCI based on ERD/ERS or SSVEP) mostly use spectral and spatial information as features whereas BCI based on ERP mostly use the temporal and spatial information. The next sections detail the corresponding feature extraction tools for these two categories of BCI.

\subsubsection{EEG signal processing tools for BCI based on oscillatory ac- tivity}

Oscillatory activity-based BCI are based on changes in power in some frequency bands, in some specific brain areas. They naturally need to exploit both the spatial and spectral information. As an example, a basic design for a motorimagery BCI would exploit the spatial information by extracting features only from EEG channels located over the motor areas of the brain, typically channels C3 for right-hand movements, $\mathrm{Cz}$ for feet movements and $\mathrm{C} 4$ for left-hand movements. It would exploit the spectral information by focusing on two frequency bands $\mu(8-12 \mathrm{~Hz})$ and $\beta(16-24 \mathrm{~Hz})$. More precisely, for a BCI that can recognise left hand MI versus right hand MI, the basic features extracted would be the average power in $\mu$ and $\beta$ bands from both channels C3 and C4. Therefore, the EEG signals would be described by only 4 features.

There are many ways to compute band power features from EEG signals $[63,64]$. However, a simple one is to first band-pass filter the EEG signal from

a given channel into the frequency band of interest, then to square the resulting signal to compute the signal power, and finally to average it over time (e.g., over a time window of $1 \mathrm{~s}$ ). This is illustrated in Figure 6 .

This basic design is unfortunately far from being optimal. Indeed, it uses only two fixed channels and two fixed frequency bands. As such, relevant information, from other channels or frequency bands, might be missing, and these two channels and bands may not be the best for the target subject. In general, better performances are obtained by using more channels [65, 66]. However, using more channels means extracting more features, thus increasing the dimensionality of the data, making the classifier optimisation more difficult. To efficiently deal with multiple channels, one can perform 1) Feature selection to automatically select a subset of relevant features [67]; 2) Channel selection to select automatically a subset of relevant channels, among all channels available [66] or 3) Spatial Filtering to combine several channels into a single one, from which features will be extracted. For EEG signal classification, spatial filtering is one of the most efficient and popular approaches [68]. It defines a smaller number of new channels as linear combinations of the original ones: 


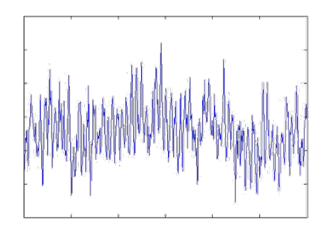
1- Raw EEG signal in C3
(left sensorimotor cortex)

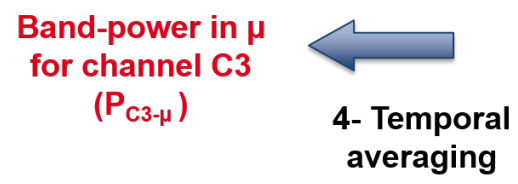

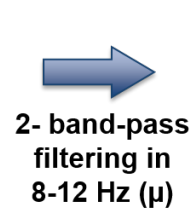

\section{3.}

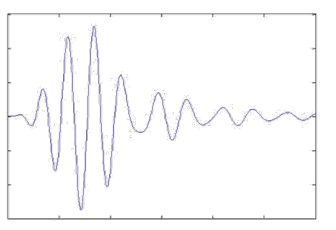

- Power estimation (squaring)

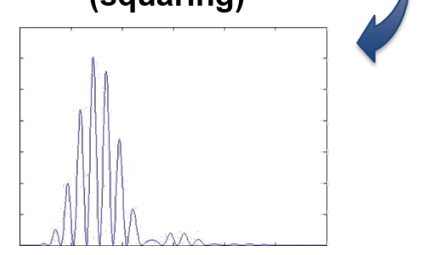

Figure 6: Signal processing steps to extract band power features from raw EEG signals. The EEG signal displayed here was recorded during right hand motor imagery (the instruction to perform the imagination was provided at $t=0 \mathrm{~s}$ on the plots), on electrode C3 (located over the left sensorimotor cortex), for 1 second. The contralateral ERD during imagination is here clearly visible in the last half of the signal. Indeed, the signal power in channel C3 (left motor cortex) in 8-12 Hz clearly decreases while the subject imagines a right hand movement.

$$
\tilde{x}(t)=\sum_{i} w_{i} x_{i}(t)
$$

with $\tilde{x}(t)$ the spatially filtered signal, $x_{i}(t)$ the EEG signal from channel $\mathrm{i}$ at time $t$ and $w_{i}$ the weight given to that channel in the spatial filter. Spatial filtering is not only useful because it reduces the dimensionality of the problem, but also because it can reduce spatial blur due to volume conduction as mentioned in Section 3.1.

For BCI based on oscillatory EEG activity, the gold-standard in spatial filtering is a supervised algorithm (i.e. an algorithm that uses training data labelled with their classes) known as the Common Spatial Patterns (CSP) algorithm $[68,69]$. Informally, the CSP algorithm finds spatial filters $w$ that maximise the variance of the filtered signal for one class and minimise it for the other class. Since the variance of a band-pass signal is actually the band-power of this signal, this means that CSP finds spatial filters that lead to maximally discriminant band-power features since their values are maximally different between classes. In [70], it was shown that "for two-class paradigms CSP maximizes an approximation of mutual information of extracted EEG components and class labels". As such, CSP is particularly useful for BCI based on oscillatory activity, in which the relevant features are band-power features. As an example, for BCI based on motor imagery, EEG signals are typically filtered in the $8-30 \mathrm{~Hz}$ band before being spatially filtered with CSP [69]. Indeed this band contains both 
the $\mu$ and $\beta$ rhythms. Formally, CSP uses the spatial filters $w$ which extremise (i.e. maximise or minimise) the following function:

$$
J(w)=\frac{w X_{1} X_{1}^{T} w^{T}}{w X_{2} X_{2}^{T} w^{T}}
$$

which is equivalent to extremising

$$
J_{C S P}(w)=\frac{w C_{1} w^{T}}{w C_{2} w^{T}}
$$

where $T$ denotes transpose, $X_{i}$ is the training band-pass filtered signal matrix for class $i$ (with the samples as columns and the channels as rows) and $C_{i}$ an estimate of the spatial covariance matrix from class $i$. In practice, the covariance matrix $C_{i}$ is usually estimated as the average covariance matrix of each trial from class $i$ from the training set [68]. In this equation, $w X_{i}$ is the spatially filtered EEG signal from class $i$, and $w X_{i} X_{i}^{T} w^{T}$ is thus the variance of the spatially filtered signal, i.e., the band-power of the spatially filtered signal. Therefore, extremising $J_{C S P}(w)$, i.e., maximising and minimising it, indeed leads to spatially filtered signals whose band-power is maximally different between classes. $J_{C S P}(w)$ happens to be a generalized Rayleigh quotient. Therefore, extremising it can be solved by Generalized Eigen Value Decomposition (GEVD). The spatial filters $w$ that maximise or minimise $J_{C S P}(w)$ are thus the eigenvectors corresponding to the largest and lowest eigenvalues, respectively, of the GEVD of matrices $C_{1}$ and $C_{2}$. Typically, 6 filters (i.e., 3 pairs), corresponding to the 3 largest and 3 lowest eigenvalues are used. Once these filters have been computed, a CSP feature $f$ is defined as follows:

$$
f=\log \left(w C w^{T}\right)
$$

i.e., the features used are simply the band power of the spatially filtered signals. The use of CSP is illustrated in Figure 7. In this figure, the signals spatially filtered with CSP clearly show differences in variance (i.e., in band power) between the two classes, hence ensuring high classification performances. It is worth mentioning that the spatial filters $w$ are only filters, and not the scalp topographies of the corresponding brain sources. As such the spatial filter weights should not be interpreted from a neurophysiological point of view [71]. Indeed, spatial filters can be seen as an unmixing or inverse model, whereas the forward or mixing model is the one that should be neurophysiologically interpreted. To go from the spatial filters (backward model) to the spatial patterns (forward model), i.e., to the scalp topography of the corresponding brain sources, one should work with the spatial patterns matrix $A=\left(W^{-1}\right)^{T}$ with $W$ the matrix with the $w$ as columns $[68,71]$. The spatial patterns, i.e., the columns of $A$, are the scalp topographies of the brain sources targeted by the spatial filters $W$.

As mentioned above, CSP is a gold standard algorithm for BCI based on oscillatory activity. Nonetheless, CSP has been shown not to be very robust to noise, non-stationarities and artefacts and to be prone to overfitting (i.e., 


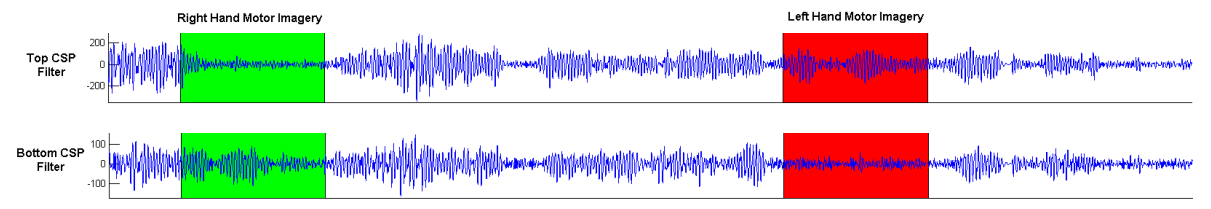

Figure 7: EEG signals spatially filtered using the CSP (Common Spatial Patterns) algorithm. The first spatial filter (top filter) maximises the variance of signals from class "Left Hand Motor Imagery" while minimising that of class "Right Hand Motor Imagery". It corresponds to the largest eigenvalue of the GEVD. The last filter (bottom filter) is the opposite: it maximises the variance of class "Right Hand Motor Imagery" while minimising that of class "Left Hand Motor Imagery" (it corresponds to the lowest eigenvalue of the GEVD). This can be clearly seen during the periods of right or left hand motor imagery, in green and red respectively.

it may not generalise well to new data) when little training data is available $[70,72,73]$. As such, many CSP variants have been designed to make it more robust to noise and non-stationarities [74, 75], or to enable it to optimise both spatial and spectral filters at the same time [76, 77].

\subsubsection{EEG signal processing tools for BCI based on event related potentials}

Like BCI based on oscillatory activity, BCI based on ERP can exploit the spatial information in EEG signals. This is typically done by focusing mostly on some specific electrodes (i.e., by extracting features only for these electrodes), e.g. for the P300, by using only electrodes located over the parietal lobe, where the P300 is known to originate. As an example, Krusienski et al. recommend to use a set of 8 channels, in positions Fz, Cz, P3, Pz, P4, PO7, Oz, PO8 [78].

Once the relevant spatial information has been identified, using, for instance, only the electrodes mentioned above, features can be extracted for the signal of each of them. For ERP in general, including the P300, the features generally exploit the temporal information of the signals, i.e., how the amplitude of the EEG signal varies with time. This is typically achieved by using the values of preprocessed EEG time points as features. More precisely, features for ERP are generally extracted by 1) low-pass or band-pass filtering the signals (e.g., in 1-12 $\mathrm{Hz}$ for the P300), 2) downsampling the filtered signals, in order to reduce the number of EEG time points and thus the dimensionality of the problem and 3) gathering the values of the resulting EEG time points from all considered channels into a feature vector that will be used as input to a classifier. This process is illustrated in Figure 8, for feature extraction from channel $\mathrm{Pz}$ in a P300-based BCI experiment.

With ERPs the number of features is usually quite large, with many features 


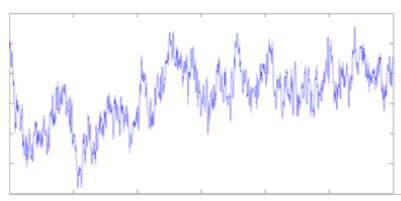

1- Raw EEG signal in

$\mathrm{Pz}$

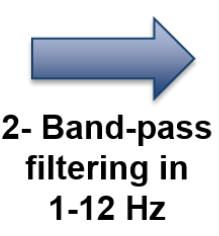

1-12 Hz

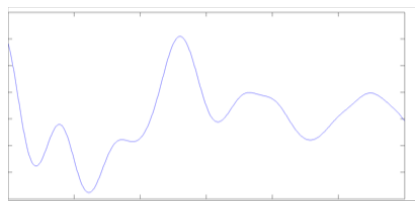

3- Down sampling (1 point out of 64)

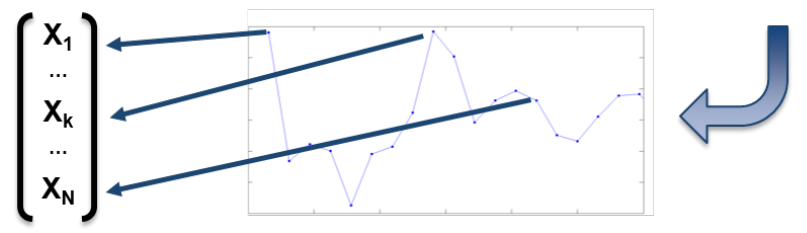

Figure 8: Typical process to extract features from a channel of EEG data for a P300-based BCI design. On this picture we can see the P300 becoming more visible with the different processing steps.

per channel and many channels used. As such, tools such as feature selection or spatial filtering can also benefit ERP-based BCI design. Regarding spatial filtering, some groups have proposed spatial filters dedicated to the classification of ERPs, leading to maximally discriminant ERP features [79, 80]. One of them is the $\mathrm{xDAWN}$ spatial filter [80]. This spatial filter aims at maximising the signal-to-signal-plus-noise-ratio. Informally, this means that xDAWN aims at enhancing the ERP response, at making the ERP more visible in the middle of the noise. Formally, xDAWN finds spatial filters that maximise the following objective function:

$$
J_{\mathrm{xDAWN}}=\frac{w A D D^{T} A^{T} w^{T}}{w X X^{T} w^{T}}
$$

where $A$ is the time course of the ERP response to detect for each channel (estimated from data, usually using a Least Square estimate) and $D$ is a matrix containing the time triggers of the target stimuli evoking the ERP. In this equation, the numerator represents the signal, i.e., the relevant information to enhance. Indeed, $w A D D^{T} A^{T} w^{T}$ is the power of the time course of the ERP responses after spatial filtering. On the contrary, in the denominator, $w X X^{T} w^{T}$ is the variance of all EEG signals after spatial filtering. Thus, it contains both the signal (the ERP) plus the noise. Therefore, maximising $J_{\mathrm{xDAWN}}$ actually maximises the signal, i.e., it enhances the ERP response, and simultaneously minimises the signal plus the noise, i.e., it makes the noise as small as possible [80]. This has indeed been shown to lead to much better ERP classification performance, especially when little training data was available. 


\subsection{Pattern recognition}

The pattern recognition stage aims at predicting a value from which infering the user's current brain activity pattern. It uses as input some features or patterns extracted from brain signals such as the power in specific frequency bands or a smoothed signal segment (see 3.2). Predicting a discrete variable corresponds to a classification task while predicting a continuous variable corresponds to a regression task.

Classification consists first in learning an efficient function $f$ from a training set of $N$ labelled examples $\left\{\left(\mathbf{d}_{1}, c_{1}\right),\left(\mathbf{d}_{2}, c_{2}\right), \ldots,\left(\mathbf{d}_{N}, c_{N}\right)\right\}$ where $\mathbf{d}_{n}$ is a feature vector and $c_{n}$ is its associated class, to allow to assign a new feature vector $\mathbf{d}$ to a unique class $c=f(\mathbf{d})$ among $C$. When $C$ only has 2 elements, the classification is binary. Numerous machine learning techniques, such as support vector machines, neural networks or logistic function, can perform this task. If $C$ contains more than 2 classes, there are two possible approaches to obtain a classification function $[81,82]$. The first approach consists in directly estimating the class using multiclass techniques such as decision trees, multilayer perceptrons, naive Bayes classifiers or k-nearest neighbours. The second approach consists in decomposing the problem into several binary classification problems. This decomposition can be done in different ways using i) one-against-one pairwise classifiers [83, 84], ii) one-against-the rest (or one-against-all) classifiers [84], iii) hierarchical classifiers similar to a binary decision tree and iv) multilabel classifiers $[85,84]$. In the later case, a distinct subset of $L$ labels (or properties) is associated to each class. The predicted class is identified according to the closest distance between the predicted labels and each subset of labels defining a class.

The performance of the classification function $f$ can be evaluated using different statistical measures. The most popular measure is the accuracy which measures the percentage of correct recognition. The accuracy is sensitive to the number of classes. The Cohen's Kappa coefficient $\kappa$ is a performance measure which takes the number of classes into consideration. However, these measures do not distinguish types of error. Indeed, when the classifier assigns a feature vector (or pattern) to class $\mathrm{A}$ whereas it actually belongs to class $\mathrm{B}$, this error has the same cost as the opposite error. In many applications, these two types of error (namely type I and II in statistical hypothesis testing) do not have the same impact. As an example, if the detection of an ERP triggers the writing of a letter, it can be more acceptable for the system not to write a letter but instead to wait for more data in order not to write an erroneous letter.

To get a detailed measure of types of error, it is possible to build a confusion matrix. This 2D-table reports how many patterns from each class (true label) have been assigned to which class (estimated label). The occurrences in the diagonal correspond to the correct assignments. Occurrences outside the diagonal correspond to different types of errors. If it is more important to take care of the performance of a specific class (named $\mathrm{P}$ as positive), it is possible to compute the recall (or sensitivity) and the precision of the classification function for this 
class. Correctly classified patterns of this class are called True Positives (TP) because the specific class to look at is identified as the positive one, and the other classes are identified as the negative ones. The recall is computed as the ratio of $\mathrm{TP}$ to the total number of patterns of class $\mathrm{P}$. This measure indicates how good the function is at identifying patterns of class P. Nevertheless, a good value can be due to a biased classifier which excessively assigns any pattern to class P. So, it is also useful to measure the precision which corresponds to the ratio of TP to the number of patterns assigned to class P. Patterns incorrectly assigned to class $\mathrm{P}$ are called False Positives (FP). Patterns of class P assigned to another class are called False Negative (FN) and patterns of other classes than P not assigned to class P are called True Negatives (TN). In theory, both the recall and the precision rates are useful. The F-score combines these two measures in one.

Here is a summary of the statistical measures presented above:

- accuracy $=(T P+T N) /(T P+F P+F N+T N)$;

- $\kappa=$ (accuracy - probability of random agreement)/(1 - probability of random agreement);

- recall (or sensitivitxy) $=T P /(T P+F N)$ (or true positive rate);

- specificity $=T N /(F P+T N)$ (or true negative rate);

- precision $=T P /(T P+F P)$;

- $\mathrm{F}$-score $=\frac{2 \times \text { recall } \times \text { precision }}{\text { recall }+ \text { precision }}$.

In practice, it is unfortunately frequent that the recall and the precision cannot both be close to one. Thus, according to the application, it is sometimes better to favour a high recall (e.g. detection of ErrP, see section 2.3) or a high precision (e.g. detection of ERP). The Receiver Operating Characteristic (ROC) curve is used to observe the effect of the decision threshold on the sensitivity and specificity. The surface of this curve is also used as an indicator to evaluate classifier performance and to compare classifiers. Indeed, many different machine learning techniques exist for classification. The most used in BCI are the Linear Discriminant Analysis (LDA) and the Support Vector Machines (SVM), for their capabilities to obtain a robust classifier i.e. efficient with new patterns. LDAs are simple linear models which do not require any hyperparameter [20]. SVM are linear or non-linear models, depending on the selected kernel, that maximise the distance between the decision boundary and the patterns of each class [20]. Robustness, or generalisation capability, is an important issue. The classification function learned during the calibration phase must be efficient with new patterns. Thus, the classification function is evaluated using the statistical measures presented above on a subset of labelled patterns acquired for calibration. If the dataset is large, the evaluation can be done using only one subset. When it is small, a cross-validation procedure is used for which the labelled dataset is split into $N$ folds (e.g. $N=10$ ) [86]. Then the parameters 
are computed using $N-1$ folds and the classification function is evaluated on the remaining fold. The procedure is repeated 10 times switching the fold used for evaluation. This procedure is only used to estimate the performance of the classifier and avoid overfitting (i.e. a classification function which is good for already known patterns but does not generalise to new patterns). In the end, the classification function is estimated using the entire set of training data. A regularisation term can be added into the cost function to increase the robustness of the classification function [87].

Up to now in EEG-based BCI, only a few studies have considered a regression task, i.e. the prediction of a continuous variable, since the low signal-to-noise ratio of EEG signals hinders the prediction of a precise value. The process is quite similar to a classification task. The parameters of a predicting linear or non linear model are learned from a training set where the desired value is continuous. Thus, many machine learning methods have a regression version, e.g. linear regression, multilayer perceptron with a single linear output unit, support vector regression, etc. Nevertheless, different and specific performance measures should be used to assess regression algorithms such as the relative absolute error or the mean square error. These measures estimated the errors between the predicted value and the real one. A regularisation term can also be added to the error function to reduce overfitting. As an illustration, regression as been used in some BCI works aiming at predicting the kinematics of upper [88] or lower limbs [89] from EEG signals.

Currently, a good BCI performance is essentially defined as a high classification accuracy. As discussed above there are various measures associated to the performance of a classifier such as the surface under the ROC curse, the F-score or the Kappa value [90]. But other measures are associated to the final task such as the accomplishment (success/fail) or speed (duration to reach a target, reaction time, Information Transfer Rate, characters per minute) [91]. Thus, although many metrics exist, one must question what they really mean for the user? Is she/he able to keep them stable or to improve them? Does she/he use a trick to control the BCI such as muscular artifacts or other brain areas than the ones expected? Is she/he able to use the BCI for a long time, without a long calibration time, without fatigue and with satisfaction? Therefore, numerous metrics beyong statistical measures should also be considered to evaluate a BCI system, e.g., measures including the users experience and preferences as measures with questionnaires.

\subsection{Feedback}

Designing a reliable BCI requires to consider its two main components, the user and the machine. BCI should be seen as a co-adaptive system [6], with the user learning to produce EEG patterns that can be recognised by the machine,

and the machine learning to recognise such patterns. Indeed, BCI control is known to be a skill that needs to be learned and mastered by the user [6, 22]. 
This is notably essential for spontaneous BCI, that is, BCI based on spontaneously generated brain activity patterns (e.g., motor imagery-based BCI). Indeed, ERP-based BCI require very little user training [92]. The fact that spontaneous BCI control is a skill means that 1) the BCI performances of a user become better with practice and thus that 2) the user needs to learn how to produce stable, clear and distinct brain activity patterns to successfully control a BCI. A key and necessary element for successful learning of spontaneous BCI control skills is the feedback provided by the machine to the user about his/her brain activity, i.e., about the mental command recognised by the BCI $[22,93,94]$. Typically, this feedback takes the form of a visual cursor or a bar moving on screen according to the recognised brain activity pattern (see Figure $9)$.
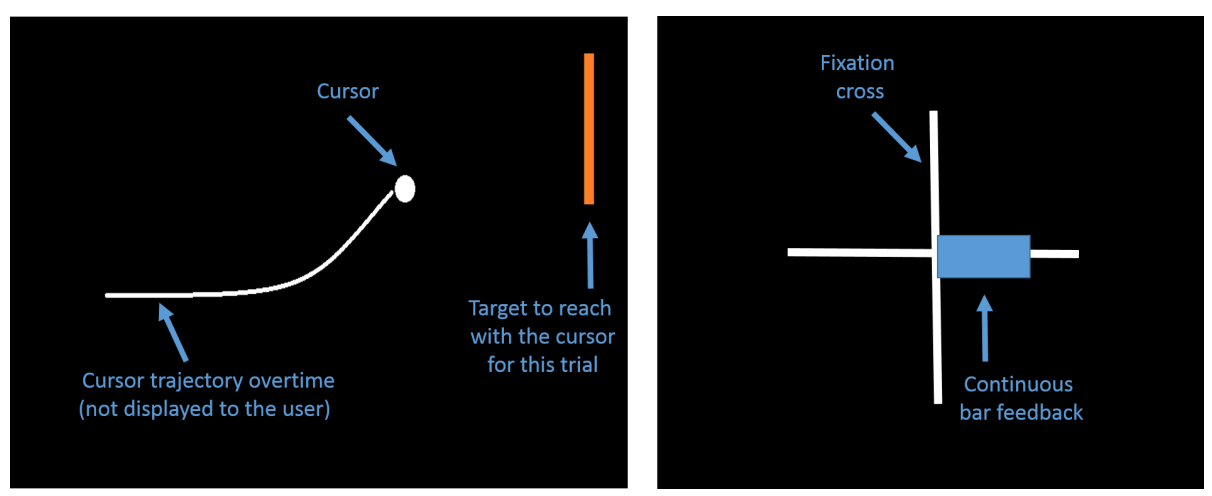

Figure 9: Examples of BCI feedback for BCI based on sensorimotor rhythms. Left: continuous cursor feedback, the cursor continuously moving from left to right, its vertical position being modulated by the sensorimotor rhythms amplitude [7]. Right: bar feedback, a bar is continuously extended towards the left or right, depending on whether the recognised EEG pattern was left or right hand movement, respectively. The bar length is proportional to the absolute classifier output [37].

However, how to train users to control BCI has been rather scarcely studied in the BCI literature so far, and the best way to train users to master BCI control skills is still unknown $[28,22]$. It is estimated that, with current systems, 10 to $30 \%$ of BCI users cannot reach BCI control at all (so-called BCI deficiency) [28]. Currently used standard training tasks and feedback are extremely simple, based on heuristics, and provide very little information. It has been shown that such standard training approaches are most likely very suboptimal with respect to general human learning and education principles [93]. For instance, typical BCI feedback is only corrective i.e., it only informs the user whether he/she performed the mental tasks correctly, whereas human learning principles recommend to provide an explanatory feedback, indicating what was right or wrong about the task performed. Therefore, there is still a need for further 
research efforts to design an ideal BCI feedback and training environment [33].

\section{Applications}

Once the BCI is able to recognise the user's brain activity patterns, it can be used to send commands to an application. Below we present some of the main applications in which BCI can be used.

\subsection{Spellers}

Several BCI systems, called "spellers" have been proposed for typing sentences without muscular control, thus eliciting communication for severely disabled patients. The most successful of these spellers is called the P300 speller [10]. The P300 speller has the advantage not to require any training on the part of the user, whose task is to concentrate his/her attention on the symbol he/she wants to type. The P300 speller can predict the symbol on which the user's attention is focussing, because flashing this symbol elicits an ERP in the EEG, the so-called P300 wave. Figure 10 illustrates the P300 speller principle.
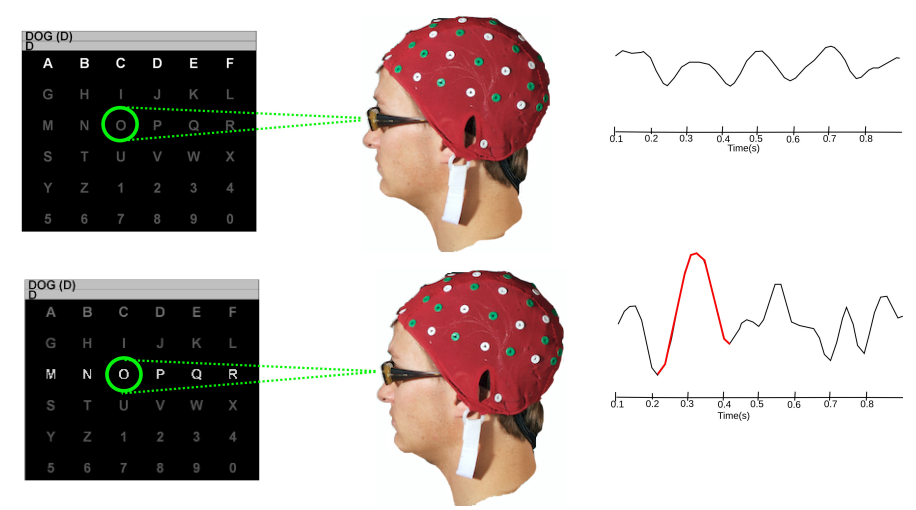

Figure 10: This user wants to type the letter "O": he counts the number of times it flashes on the screen, as in the lower picture. Each flash of the target letter creates an Evoked Response Potential, the P300 wave, time-locked to the flash display.

The BCI system has to be calibrated to detect the P300 wave from the background EEG (see section 2.3). To accelerate the throughput, keyboard symbols are organized in groups that flash simultaneously. The original P300 had a row-column flashing strategy [10], but groups can be arranged in other patterns, e.g. to minimize the probability of a given symbol flashing twice in 
a row [95]. Indeed, double flashes are both uncomfortable for the user, and produce a reduced P300 evoked response for the second flash.

The way a symbol is flashed generally consists of increasing its visual contrast with the background, and also increasing its size. Recent findings by Jin et al. show that the P300 signal-to-noise can be increased by replacing the symbols with pictures such as famous faces during the flashing interval [96].

The signal-to-noise ratio of a P300 wave is low, so sequences of flashing symbols must be repeated to make its detection possible. Each group of symbols is generally flashed several times. Besides, users are generally asked to count the number of flashes of their desired symbol in order to engage their attention. Modern P300 systems now use early stopping of the flashes as soon as a reliable decision has been made [97]. Early stopping increases the throughput, while improving users' motivation, whose focussed attention is rewarded by a faster decision $[98,99]$. In spite of the progress made in this application, it can take several seconds for a user to type a symbol with the P300 speller. Word prediction engines can be combined with the speller, to make the typing more efficient [100].

Of course, the P300 spellers can be adapted to any task relying on a choice among several elements. BCI games have been developed using this principle (see section 4.5).

A few other BCI spellers are based on other neurophysiological features than the P300. The commercial Intendix system http://www.intendix.com/ can be operated either with a P300 or an SSVEP. The Hex-O-Spell [101] uses self-paced MI features to control a rotating arrow, allowing to point to the desired letter (in two steps, each with only 6 choices).

Flashing, or even displaying symbols on a screen may not be appropriate for all users, say in the case of visual deficiencies [102]. Auditory P300 spellers have been developed, whose throughput is generally lower than for visual P300 spellers, because less symbols can be presented simultaneously to the user [103].

When hardware becomes easier to handle and to set up, BCI spellers could become commonly used by individuals with severe neuromuscular disabilities, such as amyotrophic lateral sclerosis (ALS). Several clinical studies have indeed demonstrated the usability of the spellers [104, 99], for patients who are able to maintain a sustained attention [105], and regular home use has been reported [106].

\subsection{Motor substitution}

A neuroprosthesis is a prosthesis attached to the (central or peripheral) nervous system [107]. A prosthesis is a device for improving or replacing a defective organ or a missing body part. Auditory and visual prostheses are neuroprostheses that build an alternative pathway from stimuli to the brain. In this section we will focus on motor prostheses that build an alternative pathway from brain signals or electrical nerve impulses to an artificial limb or to muscles (using functional electrical stimulation). In case of spinal cord injury, amyotrophic lateral sclerosis or brainstem stroke, the pathway from brain to muscles is interrupted before 
the nerve fibers. The activity of the motor cortex is then analysed to decode the intention of movement. Using multielectrode arrays placed invasively in the primary motor cortex, it is possible to decode the direction of movement from the activity of a neural population [108] and send commands to a robotic limb or an exoskeleton to reach and grasp a target [109]. Electrocorticography electrodes placed on the cortex are also used to obtain a good enough quality of signals, less invasively than microelectrode arrays which can cause brain tissue damage. Non-invasive signals such as EEG are also used in neuroprosthetics [12] although the spatial resolution is low and the signals are noisy. Thus, P300 wave and SSVEP can be used for a control task [50]. But ERD is most commonly used to detect a desynchronisation in various parts of the cortex meaning that a specific mental task is performed. As an example, each mental task can be translated into a command for a wheelchair in a shared-control with an obstacle avoidance system [110,111]. Motor imagery tasks are often used to control neural prosthesis or to trigger a functional electrical stimulation system $[112,113]$. It should be noted, however, that designing neuroprostheses for the lower limb is still a challenge [114], notably due to the lack of tactile or proprioceptive feedback to the user. Therefore, current efforts are focussed on adding stimulations in the somatosensory cortex or on the skin of user to provide him/her with tactile feedback and therefore to obtain a complete actionperception loop.

\subsection{Stroke rehabilitation}

A stroke (cerebral infarction or cerebral haemorrhage) is a severe common brain disorder which causes many situations of disability. Stroke is the second leading cause of death and the third leading cause of disability-adjusted life-years (DALYs) worldwide [115]. Its incidence has increased over the last two decades. One year after the accident, $30 \%$ of stroke patients do not survive. $60 \%$ of the survivors recover functional independence while about $40 \%$ are left with significant disabilities, limiting their independence in their daily lives. The majority of patients more or less recover the ability to walk, but motor control of the arm and hand often remains impaired. To limit this loss of motor control, brain-computer interfaces can be used to propose to stroke patients with residual corticomotor patterns, exercices with various feedbacks (gauge, virtual limb, robotised arm, or even functional electrical stimulation of the real limb). These exercices aim at guiding brain plasticity towards restoring efficiently and effectively motor control, or improving the control of a neuroprosthesis if not enough motor control can be recovered $[16,116,17]$. Indeed, non-invasive brain imaging techniques (fMRI, PET) and electrophysiology (TMS, EEG, MEG) can help to better understand and exploit the plasticity of the motor cortex and implement appropriate strategies for rehabilitation [117].

Recently, several groups have indeed shown that BCI-based stroke rehabilitation was possible and successful, e.g. $[118,119,120,121]$. While these are still relatively recent results, they are very promising and need to be further explored in particular to rigorously evaluate the BCI intervention versus or in 
addition to a physical therapy using for example randomized controlled trial.

\subsection{BCI and disorder of consciousness}

Another medical area in which $\mathrm{BCI}$ has recently proved to be a promising tool is for patients with Disorders of Consciousness (DoC). Indeed, following a traumatic incident or a stroke, some patients may be believed to be in a non-responsive or vegetative state (i.e., with DoC), as they show no behavioral markers of consciousness. However, some of these patients may actually be conscious or partially aware, but unable to express and show it as they are in a locked-in state. For these patients that have been erroneously diagnosed with DoC, BCI can be used to diagnose their consciousness even in the absence of any behavioural response. To do so, these patients may be asked to control a given BCI, such as a motor-imagery BCI [122], or a P300-based BCI [123]. If the patient manages to control the BCI, i.e., if the patients' EEG signals can be discriminated according to the instructions, it means the patient can cognitively respond (by performing motor imagery tasks or by attending stimulus) and therefore that he/she is conscious. If this is the case, the patient can then use the same, or another BCI to communicate with his/her environment [124]. Interested readers can refer to [125] for a review of the works in this direction and to [126] for some perspectives and challenges to tackle to bring BCI for DoC into practical clinical contexts.

\subsection{Gaming and Virtual Reality}

As mentioned earlier, potential BCI applications are far from only targeting severely motor impaired users, and can also benefit healthy users [127]. BCI can notably be seen as an exciting alternative or complementary way to control video games [128, 14, 129] and/or other virtual reality (VR) applications [13, 130]. For instance, BCI can enable a gamer to select objects in the game simply by paying attention to them $[131,132,133]$. In this case, a specific visual stimulus is displayed in overlay of each object, in order to evoke P300 or SSVEP responses. For instance, a BCI version of the popular "connect-4" game was proposed based on the P300 response [134]. In this game, the players could select the column in which to insert their token thanks to P300-based selection of the flashing columns. BCI can also be used to perform navigation tasks in game virtual environments VE $[135,136,137,138,139]$. This can be achieved for instance by performing left hand, right hand and foot MI to turn left, right and go forward respectively $[135,139,136]$ or by paying attention to flickering control buttons overlayed to the game, each button evoking a different SSVEP response and being associated to a different displacement direction in the game $[138,140]$. In all these BCI-based video games prototypes, the BCI was the sole input device. However, current BCI typically provide a rather limited number of commands (typically between 1 and 3 - with substantially lower accuracy with higher number of commands), and thus provide a limited interaction with the game. An interesting approach is therefore to use the BCI as a complementary 
input device: most of the game is controlled using a traditional input device such as a game pad, and only some actions, e.g., special powers, are controlled using the BCI. This was explored in the alphaWoW (alpha World of Warcraft) game, in which the player's avatar in the WoW game was controlled using a game pad, and this avatar can turn from a brutal bear into a fragile elf depending on the player's relaxation level, as measured in his/her EEG signals in the alpha band [141].

\subsection{Mental state monitoring and passive BCI}

Most, if not all, of the BCI-based systems and applications described so far were systems for communication and control, in which the user was voluntarily sending mental commands to the application. These types of BCI are known either as active BCI, when the user performs mental tasks (e.g., motor imagery), or as reactive BCI, when the users have to attend to stimuli (e.g., P300 or SSVEP-BCI) [15]. There is yet another category of BCI: passive BCI, for which the mental state of the user is passively estimated, without any voluntary mental command from the user, in order to adapt the application in real-time to this mental state [15]. For instance, some passive BCI can be used to monitor vigilance/alertness levels of operators performing repetitive tasks, such as x-ray luggage controllers in airports [142]. Detecting lapses of attention reduces the risk of missing threats, and can be used to propose a break to tired operators. Another example would be a passive BCI used in a car, to detect emergency braking intentions. Emergency braking intentions can indeed be detected in EEG signals before the actual physical braking, hence making it possible to stop the car earlier, potentially avoiding an accident [143]. Note that in this specific case, the used BCI design and signal processing approach are similar to that of active BCI based on movement onset detections. However, in the case of emergency braking intentions, the user does not have to do anything specific to control the BCI: he/she is driving his/her car normally, while his/her brain activity is passively monitored to detect emergency braking intention. There are actually increasingly more passive BCI application prototypes being proposed, and which were reviewed in $[144,15]$. Indeed, a number of interesting mental states can be estimated, more-or-less reliably, in EEG signals, such as mental workload (i.e., pressure on the user cognitive resources, e.g., working memory) $[145,146]$, attention $[142,147]$ and, though less accurately, emotions [148], among others [149]. Being able to monitor continuously such mental states from EEG signals makes it possible to design applications that adapt to such mental states, to provide an improved user experience [150] or simply to assess the ergonomic qualities of the system [151, 152], among many potential applications. 


\section{Hardware and software platforms for BCI}

In addition to selecting the specific brain signals, signal processing algorithms and feedback to use for the targeted BCI-based application, a BCI designer must also make practical and technical choices regarding hardware (choice of EEG sensors) and software (choice of real-time BCI software platform).

At the hardware level, one must consider what type of EEG electrodes and associated amplifier to use. In particular, EEG electrodes can either be gelbased, wet or dry. Gel-based electrodes use conductive gel or paste between the EEG sensors and the scalp, in order to reduce impedance and obtain good quality signals [153] (see also Section 2.1). Most BCI systems described in the literature use gel-based electrodes with medical-grade EEG amplifiers, which is the configuration providing the best signal quality so far. However, the use of gel makes the system long to setup and rather cumbersome for the user, who will have to wash his/her hair after each use. Alternative EEG sensors, which do not require gel, are currently being developed. For instance, wet electrodes only require to soak the sensor in a liquid solution, or dry electrodes do not require any gel nor solution, and can thus be used immediately [154]. Several dry EEG sensors have been recently developed or are currently being developed with interesting results $[155,156]$. These dry sensors are now able to acquire good quality EEG, and are much faster to setup than gel-based systems, at the cost of still more sensitivity to environmental noise and artifacts, notably artefacts due to the user's motion. These last years have also witnessed the advent of consumer grade EEG devices, such the Neurosky Mindwave (http://neurosky . com/) or the Emotiv Epoc (www.emotiv.com). These EEG devices are cheap (e.g., about $\$ 300$ for the Emotiv Epoc), do not use gel (they use either wet or dry EEG sensors) and have an attractive design. They have therefore contributed to making BCI design and use more popular and widespread. However, these devices provide lower quality EEG signals [157], and are notably sensitive to facial EMG artefacts due to their use of multiple electrodes located near the neck, on the forehead or over temporal locations [158]. Using them therefore requires taking extra care to ensure that the signals are not corrupted with undesired EMG artefacts.

On the software side, there are now a handful of mature and open-source software solutions for the design of real-time EEG-based BCI, see [159] for a recent overview. The two most widely used are BCI2000 [160] (http://bci2000.org) and OpenViBE [161] (http://openvibe.inria.fr). These two software platforms come with a number of ready-made BCI scenarios (motor imagery-based BCI, P300-speller, etc.), a number of widely used EEG signals processing and classification algorithms, and support an increasing number of EEG acquisition devices. These two software are now both open-source, which makes them easily customisable and extensible, with a large community of users. An interesting initiative to mention is also the TOBI interfaces, which are software components to enable different BCI software platforms to be connected to each other and thus to benefit from each other functionalities [162]. 


\section{Discussion and Conclusion}

Overall, EEG-based BCI is currently a very active and dynamic research topic, with hundreds of labs involved, and with many promising potential outcomes in the future. Nonetheless, EEG-based BCI are still far from being free of limitations. In particular, they still suffer for a relatively poor reliability $[28,6]$, as they often erroneously interpret the user's mental commands, and still do not work for a substantial portion of users. This may prevent them from competing with alternative input devices, e.g., eye-trackers for motor impaired users who retain some eye movements. The usability of BCI must still be improved, with more comfortable and practical EEG caps and electrodes, shorter calibration, and increased support for operating and exploring these systems which are generally not "plug-and-play". The reliability issue, long calibration times and lack of comfort are issues that currently prevent BCI from actually reaching the mainstream video game market, as the gamer population is very has high expectations regarding speed, accuracy and ease-of-use of their gaming devices [163].

Many research efforts are still needed to bring EEG-based BCI outside laboratories, to users' homes, to the consumer market and to hospitals, for daily use. To improve the reliability of BCI, some interesting research directions are a) to improve machine learning algorithms by collecting very large data sets of EEG signals from multiple contexts and users, to improve BCI calibrations; b) to explore new developments in machine learning and signal processing in order to design invariant features and classifiers that could potentially greatly increase the robustness and reliability of the system [164]; and c) to improve BCI user training protocols to make them comply with human learning theories and guidelines, hence hopefully dramatically increasing users' BCI control skills [33]. Improvement in neuroscientific knowledge could also lead to the discovery of new features or new brain activity patterns than can be exploited reliably for BCI. Conversely, BCI opens the way to new neuroscientific experiments with the user in the loop [165].

With BCI becoming more mature, and increasingly more brain activity patterns being detectable in EEG signals, many new BCI-based applications could and should be explored. This is notably the case of passive BCI, which is still rather recent and thus still underexplored but very promising, for healthy users as well as for patients (e.g., for patients' mental states monitoring and automatic treatment). New applications of BCI could further boost R\&D in the area. According to a recent questionnaire sent to the BCI community, the coordination group of the 'BNCI Horizon 2020' project identified four visions [166]: i) Patients will use invasive BCIs to compensate for movement disorders; ii) Passive BCIs will enrich human-computer interaction; iii) BCIs will be commonly used as tools for basic research and iv) the use of BCI technology for rehabilitation will increase. For six application scenarios (replace, restore, enhance, supplement, improve, research tool [6]), a future use case has been re-

ported (see http://bnci-horizon-2020.eu/roadmap) respectively: unlocking the completely locked-in, neuroprosthesis, user experience in computer games, 
BCI-controlled glass, upper limb rehabilitation after stroke, tools for cognitive neurosciences. The associated roadmap specify for the six application scenarios the available knowledge in 2015, the research in progress up to 2025 and the possible state-of-the-art in 2035 (resp. speech decoder, decoding of complex movements, wearable devices \& applications, BCIs for rehabilitation at home, plug and play tools for research).

When developing BCI, it is also essential to consider the underlying ethical issues, regarding the brain activity patterns to measure and to use (e.g., should we access and reveal them? and what for?) or about how, when and whether to use them with motor impaired or healthy users (e.g., should BCI be used to enhance an individual's abilities?), among others [167]. Overall, EEG-based BCI research has very exciting research and development perspectives in the years to come.

\section{References}

[1] H. Berger. Ueber das elektroenkephalogramm des menschen. Archiv fur Psychiatrie und Nervenkrankheiten, 87:527-570, 1929.

[2] Alvin Lucier. Music for solo performer. Lovely Music, Ltd., 1982. for enormously amplified brain waves and percussion - originally composed in 1965.

[3] Pierre Henry. Mise en musique du corticalart de Roger Lafosse. Philips "Prospective 21e sicle", 1971. Vinyl LP.

[4] J. J. Vidal. Toward direct brain-computer communication. Annual Review of Biophysics and Bioengineering, 2:157-180, 1973.

[5] J.R. Wolpaw, N. Birbaumer, D.J. McFarland, G. Pfurtscheller, and T.M. Vaughan. Brain-computer interfaces for communication and control. Clinical Neurophysiology, 113(6):767-791, 2002.

[6] J. R. Wolpaw and E. W. Wolpaw. BCI: Principles and Practice. Oxford University Press, 2012.

[7] J. R. Wolpaw, D. J. McFarland, G. W. Neat, and C. A. Forneris. An EEGbased brain-computer interface for cursor control. Electroencephalography and clinical neurophysiology, 78:252-259, 1991.

[8] G. Pfurtscheller, D. Flotzinger, and J. Kalcher. Brain-computer interfacea new communication device for handicapped persons. journal of microcomputer application, 16:293-299, 1993.

[9] N. Birbaumer, N. Ghanayim, T. Hinterberger, I. Iversen, B. Kotchoubey, A. Kübler, J. Perelmouter, E. Taub, and H. Flor. A spelling device for the paralysed. Nature, 398:297-298, 1999. 
[10] L.A. Farwell and E. Donchin. Talking off the top of your head: toward a mental prosthesis utilizing event-related brainpotentials. Electroencephalography and Clinical Neurophysiology, 70(6):510 - 523, 1988.

[11] Bernhard Graimann, Brendan Allison, and Gert Pfurtscheller. Braincomputer interfaces: Revolutionizing human-computer interaction. Springer, 2010.

[12] J.R. Millán, R. Rupp, G. Müller-Putz, R. Murray-Smith, C. Giugliemma, M. Tangermann, C. Vidaurre, F. Cincotti, A. Kübler, R. Leeb, C. Neuper, K.-R. Müller, and D. Mattia. Combining brain-computer interfaces and assistive technologies: State-of-the-art and challenges. Frontiers in Neuroprosthetics, 2010.

[13] A. Lécuyer, F. Lotte, R.B. Reilly, R. Leeb, M. Hirose, and M. Slater. Brain-computer interfaces, virtual reality and videogames. IEEE Computer, 41(10):66-72, 2008.

[14] D Coyle, J Principe, F Lotte, and A Nijholt. Guest editorial: Brain/neuronal computer games interfaces and interaction. IEEE Transactions on Computational Intelligence and AI in Games, 5(2):77-81, 2013.

[15] T.O. Zander and C. Kothe. Towards passive brain-computer interfaces: applying brain-computer interface technology to human-machine systems in general. Journal of Neural Engineering, 8, 2011.

[16] J.J. Daly and J.R. Wolpaw. Brain-computer interfaces in neurological rehabilitation. The Lancet Neurology, 7(11):1032-1043, 2008.

[17] Kai Keng Ang and Cuntai Guan. Brain-computer interface in stroke rehabilitation. Journal of Computing Science and Engineering, 7(2):139-146, 2013.

[18] J.R. Wolpaw, G.E. Loeb, B.Z. Allison, E. Donchin, O.F. do Nascimento, W.J. Heetderks, F. Nijboer, W.G. Shain, and J. N. Turner. BCI meeting 2005-workshop on signals and recording methods. IEEE Transaction on Neural Systems and Rehabilitation Engineering., 14(2):138-141, 2006.

[19] A. Bashashati, M. Fatourechi, R. K. Ward, and G. E. Birch. A survey of signal processing algorithms in brain-computer interfaces based on electrical brain signals. Journal of Neural engineering, 4(2):R35-57, 2007.

[20] F. Lotte, M. Congedo, A. Lécuyer, F. Lamarche, and B. Arnaldi. A review of classification algorithms for EEG-based brain-computer interfaces. Journal of Neural Engineering, 4:R1-R13, 2007.

[21] A. Kübler, V.K. Mushahwar, L.R. Hochberg, and J.P. Donoghue. BCI meeting 2005-workshop on clinical issues and applications. IEEE Transactions on Neural Systems and Rehabilitation Engineering, 14(2):131-134, 2006. 
[22] C. Neuper and G. Pfurtscheller. Brain-Computer Interfaces, chapter Neurofeedback Training for BCI Control, pages 65-78. The Frontiers Collection, 2010.

[23] Pieter-Jan Kindermans, Michael Tangermann, Klaus-Robert Müller, and Benjamin Schrauwen. Integrating dynamic stopping, transfer learning and language models in an adaptivezero-training erp speller. Journal of Neural Engineering, 11(3):035005, 2014.

[24] F. Lotte. Signal processing approaches to minimize or suppress calibration time in oscillatory activity-based brain-computer interfaces. Proceedings of the IEEE, 2015.

[25] C. Guger, G. Edlinger, W. Harkam, I. Niedermayer, and G. Pfurtscheller. How many people are able to operate an EEG-based brain-computer interface (BCI)? IEEE Transactions on Neural Systems and Rehabilitation Engineering, 11(2):145-147, 2003.

[26] C. Guger, S. Daban, E. Sellers, C. Holzner, G. Krausz, R. Carabalona, F. Gramatica, and G. Edlinger. How many people are able to control a P300-based brain-computer interface (BCI)? Neuroscience Letters, 462(1):94-98, 2009.

[27] Christoph Guger, Brendan Z Allison, Bernhard Großwindhager, Robert Prückl, Christoph Hintermüller, Christoph Kapeller, Markus Bruckner, Gunther Krausz, and Günter Edlinger. How many people could use an ssvep bci? Frontiers in neuroscience, 6, 2012.

[28] B.Z. Allison and C. Neuper. Could anyone use a BCI? In Desney S. Tan and Anton Nijholt, editors, Brain-Computer Interfaces, volume 0 of Human-Computer Interaction Series, pages 35-54. Springer London, 2010.

[29] S. Brandl, J. Höhne, K.-R. Müller, and W Samek. Bringing bci into everyday life: Motor imagery in a pseudo realistic environment. In roceedings of the International IEEE/EMBS Neural Engineering Conference (NER), 2015 .

[30] M. Fatourechi, A. Bashashati, R. Ward, and G. Birch. EMG and EOG artifacts in brain computer interface systems: A survey. Clinical Neurophysiology, 118(3):480-494, 2007.

[31] F. Lotte, J. Fujisawa, H. Touyama, R. Ito, M. Hirose, and A. Lécuyer. Towards ambulatory brain-computer interfaces: A pilot study with P300 signals. In 5th Advances in Computer Entertainment Technology Conference (ACE), pages 336-339, 2009.

[32] N. Neumann and A. Kubler. Training locked-in patients: a challenge for the use of brain-computer interfaces. IEEE Transactions on Neural Systems and Rehabilitation Engineering, 11(2):169-172, 2003. 
[33] Fabien Lotte and Camille Jeunet. Towards improved bci based on human learning principles. In 3rd International Brain-Computer Interfaces Winter Conference, 2015.

[34] E. Niedermeyer and F. Lopes da Silva. Electroencephalography: Basic Principles, Clinical Applications, and Related Fields. Philadelphia, Lippincott Williams \& Wilkins, 2005.

[35] H. H. Jasper. The ten-twenty electrode system of the international federation. Electroencephalography and Clinical Neurophysiology, 10(2):371-375, 1958.

[36] G. Pfurtscheller and F. H. Lopes da Silva. Event-related EEG/MEG synchronization and desynchronization: basic principles. Clinical Neurophysiology, 110(11):1842-1857, 1999.

[37] G. Pfurtscheller and C. Neuper. Motor imagery and direct brain-computer communication. proceedings of the IEEE, 89(7):1123-1134, 2001.

[38] E.V.C. Friedrich, R. Scherer, and C. Neuper. Long-term evaluation of a 4-class imagery-based brain-computer interface. Clinical Neurophysiology, 124(5):916 - 927, 2013.

[39] J.R. Millán, J. Mouri no, M. Franzé, F. Cincotti, M. Varsta, J. Heikkonen, and F. Babiloni. A local neural classifier for the recognition of eeg patterns associated to mental tasks. IEEE transactions on neural networks, 13(3):678-686, 2002.

[40] J. Kronegg, G. Chanel, S. Voloshynovskiy, and T. Pun. EEG-based synchronized brain-computer interfaces: A model for optimizing the number of mental tasks. IEEE Transactions on Neural Systems and Rehabilitation Engineering, 15(1):50-58, 2007.

[41] Connie C. Duncan, Robert J. Barry, John F. Connolly, Catherine Fischer, Patricia T.Michie, Risto Näätänen, John Polich, Ivar Reinvang, and Cyma Van Petten. Event-related potentials in clinical research: Guidelines for eliciting, recording, andquantifying mismatch negativity, P300, and N400. Clinical Neurophysiology, 120(11):1883 - 1908, 2009.

[42] R.M. Chapman and H.R. Bragdon. Evoked responses to numerical and non-numerical visual stimuli while problem solving. Nature, 203:1155-57, 1964.

[43] John Polich. Updating p300: An integrative theory of p3a and p3b. Clinical Neurophysiology, 118(10):2128 - 2148, 2007.

[44] Ricardo Chavarriaga, Aleksander Sobolewski, and José del R Millán. Errare machinale est: The use of error-related potentials in brain-machine interfaces. Frontiers in Neuroscience, 8(208), 2014. 
[45] Gerwin Schalk, Jonathan R Wolpaw, Dennis J McFarland, and Gert Pfurtscheller. Eeg-based communication: presence of an error potential. Clinical Neurophysiology, 111(12):2138-2144, 2000.

[46] G.R. McMillan, G.L. Calhoun, M.S. Middendorf, J.H Schuner, D.F. Ingle, and V.T. Nashman. Direct brain interface utilizing self-regulation of steady-state visual evoked response. In Proceedings of RESNA, pages 693-695, 1995.

[47] F.B. Vialatte, M. Maurice, J. Dauwels, and A. Cichocki. Steady-state visually evoked potentials: Focus on essential paradigms and future perspectives. Progress in Neurobiology, 90:418-438, 2010.

[48] G. Müller-Putz, R. Scherer, C. Neuper, and G. Pfurtscheller. Steadystate somatosensory evoked potentials: suitable brain signals for braincomputer interfaces. IEEE transactions on neural systems and rehabilitation engineering, 14(1):30-37, 2006.

[49] C. Gouy-Pailler, S. Achard, B. Rivet, C. Jutten, E. Maby, A. Souloumiac, and M. Congedo. Topographical dynamics of brain connections for the design of asynchronous brain-computer interfaces. In Proc. Int. Conf. IEEE Engineering in Medicine and Biology Society (IEEE EMBC), pages 2520-2523, 2007.

[50] Y Li, J Pan, F Wang, and Z Yu. A hybrid bci system combining p300 and ssvep and its application to wheelchair control. IEEE Transactions on Biomedical Engineering, 2013.

[51] H. Cecotti. A self-paced and calibration-less ssvep-based brain-computer interface speller. IEEE Transactions on Neural Systems and Rehabilitation Engineering, 18(2):127 -133, april 2010.

[52] P. Martinez, H. Bakardjian, and A. Cichocki. Fully online multicommand brain-computer interface with visual neurofeedback using SSVEP paradigm. Comput. Intell. Neuroscience, 1, 2007.

[53] N Kosmyna and F Tarpin-Bernard. Evaluation and comparison of a multimodal combination of bci paradigms and eye tracking with affordable consumer-grade hardware in a gaming context. IEEE Transactions on Computational Intelligence and AI in Games (IEEE-TCIAIG), 5(2):150 $-154,2013$.

[54] Robert Leeb, Marcel Lancelle, Vera Kaiser, Dieter W Fellner, and Gert Pfurtscheller. Thinking penguin: Multi-modal brain-computer interface control of a vr game. IEEE Transactions on Computational Intelligence and AI in Games, 5(EPFL-ARTICLE-183369):117-128, 2013.

[55] Gert Pfurtscheller, Brendan Z Allison, Günther Bauernfeind, Clemens Brunner, Teodoro Solis Escalante, Reinhold Scherer, Thorsten O Zander, 
Gernot Mueller-Putz, Christa Neuper, and Niels Birbaumer. The hybrid BCI. Frontiers in neuroscience, 4:3, 2010.

[56] G. Müller-Putz, R. Leeb, M. Tangermann, J. Höhne, A. Kübler, F. Cincotti, D. Mattia, R Rupp, K.-R. Müller, and J. del R. Millán. Towards non-invasive hybrid brain-computer interfaces: framework, practice, clinical application and beyond. Proceedings of the IEEE, 2015.

[57] A Schlögl, C Keinrath, D Zimmermann, R Scherer, R Leeb, and G Pfurtscheller. A fully automated correction method of EOG artifacts in EEG recordings. Clinical Neurophysiology, 118(1):98-104, 2007.

[58] S. Baillet, J.C. Mosher, and R.M. Leahy. Electromagnetic brain mapping. IEEE Signal Processing Magazine, 18(6):14-30, 2001.

[59] Lei Qin, Lei Ding, and Bin He. Motor imagery classification by means of source analysis for brain-computer interfaceapplications. Journal of Neural Engineering, 1(3):135, 2004.

[60] M. Congedo, F. Lotte, and A. Lécuyer. Classification of movement intention by spatially filtered electromagnetic inverse solutions. Physics in Medicine and Biology, 51:1971-1989, 2006.

[61] D. J. McFarland, L. M. McCane, S. V. David, and J. R. Wolpaw. Spatial filter selection for EEG-based communication. Electroencephalographic Clinical Neurophysiology, 103(3):386-394, 1997.

[62] P.L. Nunez. Electric fields of the brain: the neurophysics of EEG. Oxford University Press, 2005.

[63] P Herman, G Prasad, TM McGinnity, and D Coyle. Comparative analysis of spectral approaches to feature extraction for eeg-based motor imagery classification. Neural Systems and Rehabilitation Engineering, IEEE Transactions on, 16(4):317-326, 2008.

[64] N Brodu, F Lotte, and A Lécuyer. Comparative study of band-power extraction techniques for motor imagery classification. In Computational Intelligence, Cognitive Algorithms, Mind, and Brain (CCMB), 2011 IEEE Symposium on, pages 1-6. IEEE, 2011.

[65] C. Sannelli, T. Dickhaus, S. Halder, E.M. Hammer, K.-R. Müller, and B. Blankertz. On optimal channel configurations for SMR-based braincomputer interfaces. Brain Topography, 2010.

[66] M. Arvaneh, C. Guan, K.K. Ang, and H.C. Quek. Optimizing the channel selection and classification accuracy in eeg-based bci. IEEE Transactions on Biomedical Engineering, 58:1865-1873, 2011.

[67] I. Guyon and A. Elisseeff. An introduction to variable and feature selection. Journal of Machine Learning Research, 3:1157-1182, 2003. 
[68] B. Blankertz, R. Tomioka, S. Lemm, M. Kawanabe, and K.-R. Müller. Optimizing spatial filters for robust EEG single-trial analysis. IEEE Signal Proc Magazine, 25(1):41-56, 2008.

[69] H. Ramoser, J. Muller-Gerking, and G. Pfurtscheller. Optimal spatial filtering of single trial EEG during imagined hand movement. IEEE Transactions on Rehabilitation Engineering, 8(4):441-446, 2000.

[70] M. Grosse-Wentrup and M. Buss. Multi-class common spatial pattern and information theoretic feature extraction. IEEE Transactions on Biomedical Engineering, 55(8):1991-2000, 2008.

[71] Stefan Haufe, Frank Meinecke, Kai Görgen, Sven Dähne, John-Dylan Haynes, Benjamin Blankertz, and Felix Bießmann. On the interpretation of weight vectors of linear models in multivariate neuroimaging. NeuroImage, 87:96-110, 2014.

[72] M. Grosse-Wentrup. Understanding brain connectivity patterns during motor imagery for brain-computer interfacing. In Advances in neural information processing systems (NIPS) 21, 2009.

[73] B. Reuderink and M. Poel. Robustness of the common spatial patterns algorithm in the BCI-pipeline. Technical report, HMI, University of Twente, 2008 .

[74] F. Lotte and C.T. Guan. Regularizing common spatial patterns to improve BCI designs: Unified theory and new algorithms. IEEE Transactions on Biomedical Engineering, 58(2):355-362, 2011.

[75] W Samek, M Kawanabe, and K Muller. Divergence-based framework for common spatial patterns algorithms. IEEE Reviews in Biomedical Engineering, 2014.

[76] KK Ang, ZY Chin, C Wang, C Guan, and H Zhang. Filter bank common spatial pattern algorithm on bci competition iv datasets $2 \mathrm{a}$ and $2 \mathrm{~b}$. Frontiers in Neuroscience, 6, 2012.

[77] S. Lemm, B. Blankertz, G. Curio, and K.-R. Müller. Spatio-spectral filters for improving classification of single trial EEG. IEEE Trans. Biomed. Eng., 52(9):1541-1548, 2005.

[78] D.J. Krusienski, E.W. Sellers, F. Cabestaing, S. Bayoudh, D.J. McFarland, T.M. Vaughan, and J.R. Wolpaw. A comparison of classification techniques for the P300 speller. Journal of Neural Engineering, 3:299$305,2006$.

[79] U. Hoffmann, J. Vesin, and T. Ebrahimi. Spatial filters for the classification of event-related potentials. In European Symposium on Artificial Neural Networks (ESANN 2006), 2006. 
[80] B. Rivet, A. Souloumiac, V. Attina, and G. Gibert. xdawn algorithm to enhance evoked potentials: Application to brain computer interface. IEEE Transactions on Biomedical Engineering, 56(8):2035-2043, 2009.

[81] Grigorios Tsoumakas and Ioannis Katakis. Multi-label classification: An overview. Int J Data Warehousing and Mining, 2007:1-13, 2007.

[82] Gjorgji Madjarov, Dragi Kocev, Dejan Gjorgjevikj, and Sašo Džeroski. An extensive experimental comparison of methods for multi-label learning. Pattern Recognition, 45(9):3084 - 3104, 2012.

[83] Trevor Hastie and Robert Tibshirani. Classification by pairwise coupling. The Annals of Statistics, 26(2):451-471, 1998.

[84] Erin L. Allwein, Robert E. Schapire, Yoram Singer, and Pack Kaelbling. Reducing multiclass to binary: A unifying approach for margin classifiers. Journal of Machine Learning Research, 1:113-141, 2000.

[85] Thomas G. Dietterich and Ghulum Bakiri. Solving multiclass learning problems via error-correcting output codes. Journal of Artificial Intelligence Research, 2:263-286, 1995.

[86] M. W. Browne. Cross-validation methods. Journal of Mathematical Psychology, 44(1):108-132, 2000.

[87] R. Tomioka and K.-R. Müller. A regularized discriminative framework for eeg analysis with application to brain-computer interface. Neuroimage, 49(1):415-432, 2010.

[88] Trent J. Bradberry, Rodolphe J. Gentili, and José L. Contreras-Vidal. Reconstructing three-dimensional hand movements from noninvasive electroencephalographic signals. The Journal of neuroscience : the official journal of the Society for Neuroscience, 30(9):3432-3437, March 2010.

[89] Javier M Antelis, Luis Montesano, Ander Ramos-Murguialday, Niels Birbaumer, and Javier Minguez. On the usage of linear regression models to reconstruct limb kinematics from low frequency eeg signals. PLoS One, 8(4):e61976, 2013.

[90] E Thomas, M Dyson, and M Clerc. An analysis of performance evaluation for motor-imagery based bci. Journal of neural engineering, 10(3):031001, 2013.

[91] Eoin Thomas, Matthew Dyson, and Maureen Clerc. An analysis of performance evaluation for motor-imagery based BCI. Journal of Neural Engineering, 10(3), June 2013.

[92] R. Fazel-Rezai, BZ Allison, C Guger, EW Sellers, SC Kleih, and A Kübler. P300 brain computer interface: current challenges and emerging trends. Frontiers in Neuroengineering, 5(14), 2012. 
[93] F Lotte, F Larrue, and C Mühl. Flaws in current human training protocols for spontaneous brain-computer interfaces: lessons learned from instructional design. Frontiers in Human Neuroscience, 7(568), 2013.

[94] DJ McFarland, LM McCane, and JR Wolpaw. EEG-based communication and control: short-term role of feedback. Rehabilitation Engineering, IEEE Transactions on, 6(1):7-11, 1998.

[95] G. Townsend, B.K. LaPallo, C.B. Boulay, D.J. Krusienski, G.E. Frye, C.K. Hauserand N.E. Schwartz, T.M. Vaughan, J.R. Wolpaw, and E.W. Sellers. A novel p300-based brain-computer interface stimulus presentation paradigm: Moving beyondrows and columns. Clinical Neurophysiology, 121(7):1109 - 1120, 2010.

[96] Jing Jin, Brendan Z. Allison, Tobias Kaufmann, Andrea Kübler, Yu Zhang, XingyuWang, and Andrzej Cichocki. The changing face of P300 BCIs: A comparison of stimulus changes in a P300 BCI involvingfaces, emotion, and movement. PLoS ONE, 7(11), 2012.

[97] M. Perrin, E. Maby, O. Bertrand, and J. Mattout. A virtuous BCI loop: adaptive decision-making improves P300-spelling in two ways. In Proceedings of the 6th International Brain-Computer Interface Conference, 2014.

[98] E. Thomas, E. Daucé, D. Devlaminck, L. Mahé, A. Carpentier, R. Munos, M.Perrin, E. Maby, J. Mattout, T. Papadopoulo, and M. Clerc. CoAdapt P300 speller: optimized flashing sequences and online learning. In Proceedings of the 6th International Brain-Computer Interface Conference, 2014.

[99] B.O. Mainsah, L.M. Collins, K.A. Colwell, E.W. Sellers, D.B. Ryan, and K. Caves andC.S. Throckmorton. Increasing BCI communication rates with dynamic stopping towards more practical use: an ALSstudy. Journal of Neural Engineering, 12, 2015.

[100] D. B. Ryan, G. E. Frye, G. Townsend, D. R. Berry, S. Mesa-G, and E. W. Gates, N. A.and Sellers. Predictive spelling with a p300-based braincomputer interface: Increasing the rate ofcommunication. International Journal of Human-Computer Interaction, 27(1):69-84, 2010.

[101] Klaus-Robert Müller, Michael Tangermann, Guido Dornhege, Matthias Krauledat andGabriel Curio, and Benjamin Blankertz. Machine learning for real-time single-trial eeg-analysis: From brain-computer interfacing tomental state monitoring. Journal of Neuroscience Methods, 167(1):82 - 90, 2008. Brain-Computer Interfaces (BCIs).

[102] P Brunner, S Joshi, S Briskin, J R Wolpaw, H Bischof, and G Schalk. Does the 'p300' speller depend on eye gaze? Journal of Neural Engineering, $7(5): 056013,2010$. 
[103] A. Furdea, S. Halder, D.J. Krusienski, D. Bross, F. Nijboer, N. Birbaumer, and A.Kübler. An auditory (P300) spelling system for brain-computer interfaces. Psychophysiology, 46:617-625, 2009.

[104] Nathan A. Gates, Christopher K. Hauser, and Eric W. Sellers. A longitudinal study of p300 brain-computer interface and progression of amyotrophic lateralsclerosis. In Foundations of Augmented Cognition. Directing the Future of Adaptive Systems, Lecture Notes in Computer Science, pages 475-483. Springer Berlin Heidelberg, 2011.

[105] Angela Riccio, Luca Simione, Francesca Schettini, Alessia Pizzimenti, MaurizioInghilleri, Marta Olivetti Belardinelli, Donatella Mattia, and Febo Cincotti. Attention and P300-based BCI performance in people with amyotrophic lateral sclerosis. Frontiers in Human Neuroscience, 7, 2013.

[106] Eric W. Sellers, Theresa M. Vaughan, and Jonathan R. Wolpaw. A braincomputer interface for long-term independent home use. Amyotrophic Lateral Sclerosis, 2010.

[107] Aimee E Schultz and Todd A Kuiken. Neural interfaces for control of upper limb prostheses: the state of the art and future possibilities. $P M$ $R, 3(1): 55-67$, Jan 2011.

[108] A P Georgopoulos, A B Schwartz, and R E Kettner. Neuronal population coding of movement direction. Science, 233(4771):1416-9, Sep 1986.

[109] Leigh R. Hochberg, Daniel Bacher, Beata Jarosiewicz, Nicolas Y. Masse, John D. Simeral, Joern Vogel, Sami Haddadin, Jie Liu, Sydney S. Cash, Patrick van der Smagt, and John P. Donoghue. Reach and grasp by people with tetraplegia using a neurally controlled robotic arm. Nature, 485(7398):372-375, 052012.

[110] J del R Millán, F Galan, D Vanhooydonck, E Lew, J Philips, and M Nuttin. Asynchronous non-invasive brain-actuated control of an intelligent wheelchair. Conf Proc IEEE Eng Med Biol Soc, 2009:3361-4, 2009.

[111] T. Carlson and Y. Demiris. Collaborative control for a robotic wheelchair: Evaluation of performance, attention, and workload. Systems, Man, and Cybernetics, Part B: Cybernetics, IEEE Transactions on, 42(3):876-888, June 2012 .

[112] Gert Pfurtscheller, Gernot R Müller, Jörg Pfurtscheller, Hans Jürgen Gerner, and Rüdiger Rupp. 'thought'-control of functional electrical stimulation to restore hand grasp in a patient with tetraplegia. Neurosci Lett, 351(1):33-6, Nov 2003.

[113] Michele Tavella, Robert Leeb, Rudiger Rupp, and Jose Del R Millan. Towards natural non-invasive hand neuroprostheses for daily living. Conf Proc IEEE Eng Med Biol Soc, 2010:126-129, 2010. 
[114] M. Duvinage, T. Castermans, R. Jiménez-Fabían, T. Hoellinger, M. Petieau, O. Verlinden, G. Cheron, and T. Dutoit. Human walk modeled by pcpg to control a lower limb neuroprosthesis by high-level commands. Journal of Systemics, Cybernetics and Informatics, 10(3):70-80, 2012.

[115] Valery L Feigin, Mohammad H Forouzanfar, Rita Krishnamurthi, George A Mensah, Myles Connor, Derrick A Bennett, Andrew E Moran, Ralph L Sacco, Laurie Anderson, Thomas Truelsen, Martin O'Donnell, Narayanaswamy Venketasubramanian, Suzanne Barker-Collo, Carlene M M Lawes, Wenzhi Wang, Yukito Shinohara, Emma Witt, Majid Ezzati, Mohsen Naghavi, Christopher Murray, and Global Burden of Diseases, Injuries, and Risk Factors Study 2010 (GBD 2010) and the GBD Stroke Experts Group. Global and regional burden of stroke during 19902010: findings from the global burden of disease study 2010. Lancet, 383(9913):245-54, Jan 2014.

[116] Stefano Silvoni, Ander Ramos-Murguialday, Marianna Cavinato, Chiara Volpato, Giulia Cisotto, Andrea Turolla, Francesco Piccione, and Niels Birbaumer. Brain-computer interface in stroke: a review of progress. Clinical EEG and Neuroscience, 42(4):245-252, 2011.

[117] NS Ward and LG Cohen. Mechanisms underlying recovery of motor function after stroke. Archives of Neurology, 61(12):1844-1848, 2004.

[118] Girijesh Prasad, Pawel Herman, Damien Coyle, Suzanne McDonough, and Jacqueline Crosbie. Applying a brain-computer interface to support motor imagery practice in people with stroke for upper limb recovery: a feasibility study. J Neuroeng Rehabil, 7:60, 2010.

[119] Ander Ramos-Murguialday, Doris Broetz, Massimiliano Rea, Leonhard Läer, Özge Yilmaz, Fabricio L Brasil, Giulia Liberati, Marco R Curado, Eliana Garcia-Cossio, Alexandros Vyziotis, et al. Brain-machine interface in chronic stroke rehabilitation: A controlled study. Annals of neurology, 74(1):100-108, 2013.

[120] Donatella Mattia, Floriana Pichiorri, Marco Molinari, and Rüdiger Rupp. Brain computer interface for hand motor function restoration and rehabilitation. In Towards Practical Brain-Computer Interfaces, pages 131-153. Springer, 2013.

[121] Kai Keng Ang, Cuntai Guan, Kok Soon Phua, Chuanchu Wang, Longjiang Zhou, Ka Yin Tang, Gopal Joseph Ephraim Joseph, Christopher Wee Keong Kuah, and Karen Sui Geok Chua. Brain-computer interface-based robotic end effector system for wrist and hand rehabilitation: results of a three-armed randomized controlled trial for chronic stroke. Frontiers in Neuroengineering, 7(30), 2014. 
[122] Damien Coyle, Jacqueline Stow, Karl McCreadie, Jacinta McElligott, and Áine Carroll. Sensorimotor modulation assessment and brain-computer interface training in disorders of consciousness. Archives of physical medicine and rehabilitation, 96(3):S62-S70, 2015.

[123] Dorothée Lulé, Quentin Noirhomme, Sonja C Kleih, Camille Chatelle, Sebastian Halder, Athena Demertzi, Marie-Aurélie Bruno, Olivia Gosseries, Audrey Vanhaudenhuyse, Caroline Schnakers, et al. Probing command following in patients with disorders of consciousness using a braincomputer interface. Clinical Neurophysiology, 124(1):101-106, 2013.

[124] Gernot R Müller-Putz, Christoph Pokorny, Daniela S Klobassa, and Petar Horki. A single-switch bci based on passive and imagined movements: toward restoring communication in minimally conscious patients. International journal of neural systems, 23(02), 2013.

[125] Camille Chatelle, Srivas Chennu, Quentin Noirhomme, Damian Cruse, Adrian M Owen, and Steven Laureys. Brain-computer interfacing in disorders of consciousness. Brain injury, 26(12):1510-1522, 2012.

[126] Jacques Luauté, Dominique Morlet, and Jérémie Mattout. Bci in patients with disorders of consciousness: Clinical perspectives. Annals of Physical and Rehabilitation Medicine, 2015.

[127] J. van Erp, F. Lotte, and M. Tangermann. Brain-computer interfaces: Beyond medical applications. IEEE Computer, 45(4):26-34, 2012.

[128] A. Nijholt, D. Plass-Oude Bos, and B. Reuderink. Turning shortcomings into challenges: Brain-computer interfaces for games. Entertainment Computing, 1(2):85-94, 2009.

[129] David Marshall, Damien Coyle, Shane Wilson, and Michael Callaghan. Games, gameplay, and bci: The state of the art. Computational Intelligence and AI in Games, IEEE Transactions on, 5(2):82-99, 2013.

[130] Fabien Lotte, Josef Faller, Christoph Guger, Yann Renard, Gert Pfurtscheller, Anatole Lécuyer, and Robert Leeb. Combining BCI with Virtual Reality: Towards new applications and improved BCI. In Brendan Z. Allison, Stephen Dunne, Robert Leeb, José Del R. Millán, and Anton Nijholt, editors, Towards Practical Brain-Computer Interfaces, Biological and Medical Physics, Biomedical Engineering, pages 197-220. Springer Berlin Heidelberg, 2013.

[131] J. D. Bayliss. The use of the P3 evoked potential component for control in a virtual apartment. IEEE Transactions on Neural Systems and Rehabilitation Engineering, 11(2):113-116, 2003.

[132] E. Lalor, S. P. Kelly, C. Finucane, R. Burke, R. Smith, R. Reilly, and G. McDarby. Steady-state VEP-based brain-computer interface control 
in an immersive 3-D gaming environment. EURASIP journal on applied signal processing, 2005(19):3156 - 3164, 2005.

[133] Alexander Kaplan, S Shishkin, I Ganin, I Basyul, and A Zhigalov. Adapting the p300-based brain-computer interface for gaming: a review. Computational Intelligence and AI in Games, IEEE Transactions on, 5(2):141$149,2013$.

[134] Emmanuel Maby, Margaux Perrin, Olivier Bertrand, Gaëtan Sanchez, and Jérémie Mattout. Bci could make old two-player games even more fun: a proof of concept with connect four. Advances in Human-Computer Interaction, 2012:1, 2012.

[135] R. Leeb, F. Lee, C. Keinrath, R. Scherer, H. Bischof, and G. Pfurtscheller. Brain-computer communication: Motivation, aim and impact of exploring a virtual apartment. IEEE, Transactions on Neural Systems 83 Rehabilitation Engineering, 15(4):473 - 482, 2007.

[136] R. Scherer, F. Lee, A. Schlögl, R. Leeb, H. Bischof, and G. Pfurtscheller. Towards self-paced brain-computer communication: Navigation through virtual worlds. IEEE, Transactions on Biomedical Engineering, 55(2):675$682,2008$.

[137] F. Velasco-Álvarez and R. Ron-Angevin. Free virtual navigation using motor imagery through an asynchronous brain-computer interface. Presence: teleoperators and virtual environments, 19(1):71-81, 2010.

[138] J. Faller, G. Müller-Putz, D. Schmalstieg, and G. Pfurtscheller. An application framework for controlling an avatar in a desktop-based virtual environment via a software SSVEP brain-computer interface. Presence: teleoperators and virtual environments, 19(1):25-34, 2010.

[139] F. Lotte, A. Van Langhenhove, F. Lamarche, T. Ernest, Y. Renard, B. Arnaldi, and A. Lécuyer. Exploring large virtual environments by thoughts using a brain-computer interface based on motor imagery and high-level commands. Presence: teleoperators and virtual environments, 19(1):5470, 2010.

[140] J Legény, R Viciana Abad, and A Lécuyer. Toward contextual ssvep-based bci controller: Smart activation of stimuli and controls weighting. IEEE Transaction on Computational Intelligence and AI in Games, 2013.

[141] Bram van de Laar, Hayrettin Gurkok, D Plass-Oude Bos, Mannes Poel, and Anton Nijholt. Experiencing bci control in a popular computer game. Computational Intelligence and AI in Games, IEEE Transactions on, 5(2):176-184, 2013.

[142] B. Blankertz, M.Tangermann, C. Vidaurre, S. Fazli, C.Sannelli, S.Haufe, C. Maeder, L. Ramsey, I. Sturm, G. Curio, and K.R. Mueller. The berlin 
brain-computer interface: Non-medical uses of bci technology. Frontiers in Neuroprosthetics, 5, 2010.

[143] Stefan Haufe, Jeong-Woo Kim, Il-Hwa Kim, Andreas Sonnleitner, Michael Schrauf, Gabriel Curio, and Benjamin Blankertz. Electrophysiology-based detection of emergency braking intention in real-world driving. Journal of neural engineering, 11(5):056011, 2014.

[144] L. George and A. Lécuyer. An overview of research on passive braincomputer interfaces for implicit human-computer interaction. In International Conference on Applied Bionics and Biomechanics, 2010.

[145] A.-M. Brouwer, M.A. Hogervorst, J.B.F. van Erp, T. Heffelaar, P.H. Zimmerman, and R. Oostenveld. Estimating workload using eeg spectral power and erps in the n-back task. Journal of Neural Engineering, 9(4), 2012 .

[146] Christian Mühl, Camille Jeunet, and Fabien Lotte. EEG-based workload estimation across affective contexts. Frontiers in Neuroscience section Neuroprosthetics, 8:114, 2014.

[147] B. Hamadicharef, H.H. Zhang, C.T. Guan, C.C. Wang, K.S. Phua, K.P. Tee, and K.K. Ang. Learning EEG-based spectral-spatial patterns for attention level measurement. In Proceedings of the 2009 IEEE International Symposium on Circuits and Systems (ISCAS2009), pages 14651468, 2009 .

[148] C. Mühl, B. Allison, A. Nijholt, and G. Chanel. A survey of affective brain computer interfaces: principles, state-of-the-art, and challenges. BrainComputer Interfaces, pages 1-19, 2014.

[149] Jérémy Frey, Christian Mühl, Fabien Lotte, and Martin Hachet. Review of the use of electroencephalography as an evaluation method for humancomputer interaction. In Proc. of PhyCS, 2014.

[150] Anatole Lecuyer, Laurent George, and Maud Marchal. Toward adaptive vr simulators combining visual, haptic, and brain-computer interfaces. Computer Graphics and Applications, IEEE, 33(5):18-23, 2013.

[151] J. Frey, A. Appriou, F. Lotte, and M. Hachet. Estimating visual comfort in stereoscopic displays using electroencephalography: A proof-of-concept. In Proc. Interact, 2015.

[152] D. Wobrock, J. Frey, D. Graeff, J.-B. de la Rivière, J. Castet, and F. Lotte. Continuous mental effort evaluation during $3 \mathrm{~d}$ object manipulation tasks based on brain and physiological signals. In Proc. Interact, 2015.

[153] E. Niedermeyer. Electroencephalography: basic principles, clinical applications, and related fields, chapter The normal EEG of the waking adult. Lippincott Williams \& Wilkins, ISBN 0781751268, E. Niedermeyer and F. Lopes da Silva eds edition, 2005. 
[154] F. Popescu, S. Fazli, Y. Badower, B. Blankertz, and K.-R. Müller. Single trial classification of motor imagination using 6 dry EEG electrodes. PLoS ONE, 2(7):e637, 2007.

[155] Thorsten Oliver Zander, Moritz Lehne, Klas Ihme, Sabine Jatzev, Joao Correia, Christian Kothe, Bernd Picht, and Femke Nijboer. A dry eegsystem for scientific research and brain-computer interfaces. Frontiers in neuroscience, 5, 2011.

[156] Christoph Guger, Gunther Krausz, Brendan Z Allison, and Guenter Edlinger. Comparison of dry and gel based electrodes for p300 braincomputer interfaces. Frontiers in neuroscience, 6, 2012.

[157] Matthieu Duvinage, Thierry Castermans, Thierry Dutoit, M Petieau, T Hoellinger, C De Saedeleer, K Seetharaman, and G Cheron. A p300based quantitative comparison between the emotiv epoc headset and a medical eeg device. Biomedical Engineering Online, 765, 2012.

[158] D. Heingartner. Mental block. IEEE Spectrum, 46(1):42-43, 2009.

[159] Clemens Brunner, Giuseppe Andreoni, Lugi Bianchi, Benjamin Blankertz, Christian Breitwieser, Shin'ichiro Kanoh, Christian A Kothe, Anatole Lécuyer, Scott Makeig, Jürgen Mellinger, et al. Bci software platforms. In Towards Practical Brain-Computer Interfaces, pages 303-331. Springer, 2013.

[160] G. Schalk, D. J. McFarland, T. Hinterberger, N. Birbaumer, and J. R. Wolpaw. BCI2000: a general-purpose brain-computer interface (BCI) system. IEEE Transactions on Biomedical Engineering, 51(6):1034-1043, 2004 .

[161] Y. Renard, F. Lotte, G. Gibert, M. Congedo, E. Maby, V. Delannoy, O. Bertrand, and A. Lécuyer. OpenViBE: An open-source software platform to design, test and use brain-computer interfaces in real and virtual environments. Presence: teleoperators and virtual environments, 19(1):3553, 2010.

[162] Gernot R Müller-Putz, Christian Breitwieser, Febo Cincotti, Robert Leeb, Martijn Schreuder, Francesco Leotta, Michele Tavella, Luigi Bianchi, Alex Kreilinger, Andrew Ramsay, et al. Tools for brain-computer interaction: a general concept for a hybrid bci. Frontiers in neuroinformatics, 5, 2011.

[163] F. Lotte. Brain-computer interfaces for 3D games: Hype or hope? In Foundations of Digital Games, pages 325-327, 2011.

[164] DJ Krusienski, M Grosse-Wentrup, F Galán, D Coyle, KJ Miller, E Forney, and CW Anderson. Critical issues in state-of-the-art brain-computer interface signal processing. Journal of Neural Engineering, 8(2):025002, 2011. 
[165] Jérémie Mattout. Brain-computer interfaces: a neuroscience paradigm of social interaction? a matter of perspective. Frontiers in Human Neuroscience, 6(114), 2012.

[166] Clemens Brunner, Niels Birbaumer, Benjamin Blankertz, Christoph Guger, Andrea Kübler, Donatella Mattia, José del R. Millán, Felip Miralles, Anton Nijholt, Eloy Opisso, Nick Ramsey, Patric Salomon, and Gernot R. Müller-Putz. Bnci horizon 2020: towards a roadmap for the bci community. Brain-Computer Interfaces, 0(0):1-10, 0.

[167] Femke Nijboer, Jens Clausen, Brendan Z Allison, and Pim Haselager. The asilomar survey: Stakeholders' opinions on ethical issues related to brain-computer interfacing. Neuroethics, 6(3):541-578, 2013. 